\title{
A six-year audit in a rehabilitation centre with mod barthel index and FIM as outcome measures
}

\begin{abstract}
A rehabilitation audit was performed comparing admission and discharge values of FIM and Barthel Index (self report) of the last 6 years. The results indicate substantial changes in FIM and BI thus justifying the rehabilitation process. Performing an audit of the used instruments we found a small group of patients (between 6\% (post surgery patients) and $26.4 \%$ (rheumatological patients)) for whom no overall improvement could be indicated. These patients differ significantly from the other patients in reaching higher values on admission thus indicating a possible ceiling effect in FIM and in $\mathrm{BI}$
\end{abstract}

Volume 3 Issue 4 - 2018

\author{
Valach L, Selz B, Hofer K, Jann C
}

Designation University, Switzerland

Correspondence: Ladislav Valach, Designation University, Lindenstrasse 26, 3047 Bremgarten, Switzerland, Tel

+31792162192, Email ladislav.valach@swissonline.ch

Received: August 03, 2018| Published: August 31, 2018

\section{Introduction}

Current rehabilitation standards require evidence based rehabilitation procedures and a significant outcome improvement as a consequence of the implemented rehabilitation programmes. While the analyses on which medicine bases its evidence stem from prospective, control studies performed mostly by university centres with the highest methodological vigour, the routine outcome measure analyses have to be conducted using locally available resources. Additionally, it has been suggested that the demonstration of outcome improvement is complicated by many problems such as small numbers and heterogeneity in the study population. ${ }^{1,2}$ There are strategies to overcome this particular problem. Multicentre trials or meta-analyses provide some possibilities; a long-term data collection offers another one.

Selecting an outcome measure for a rehabilitation centre a broad spectrum of patients, diagnoses and treatments should be considered in addition to validity, reliability and sensitivity to the relevant changes targeted by the intervention. The measures must also be feasible to use in routine practice and the team should find them useful.

Analysing data collected over a long time span as in our case the assumptions and decisions made at the time of the planning and implementation of the outcome measures must be to some degree adhered to. There has been a high increase in the last years in publication of relevant studies dealing with the issue of outcome measure in rehabilitation that added to our knowledge on outcome measures in a substantial way. In the early nineties when our system of outcome measures was designed the therapists constructed a net of global and of specific measures of which they believed would provide a comprehensive monitoring of their goal attainment in rehabilitation. The dimensions of independence, disability and resources play an important role in most rehabilitation goals. Several global measures of independence in a range of tasks gained in popularity in the last decades. A survey of rehabilitation units in the $\mathrm{UK}^{3}$ demonstrated that the Barthel Index (BI), ${ }^{4}$ the Functional Independence Measure $(\mathrm{FIM})^{5}$ or the Functional Assessment Measure (FIM+FAM) ${ }^{6}$ are the most commonly used global measures. Sulter, Steen and De Keyser ${ }^{7}$ analysed all randomized trials between 1995 and 1998 in which the efficacy of thrombolytic, neuroprotective drugs or antithrombotic compounds was investigated and found that BI was the most often used outcome measure.

Although the BI and FIM were published a long time ago and gained wide acceptance, ${ }^{8-10}$ a series of substantial controversies were carried out in professional journals indicating number of essential shortcomings, ${ }^{11}$ such as floor and ceiling effects (BI), (see ${ }^{12}$ for ceiling effect in amputee patients; $;^{13}$ for ceiling and floor effect in stroke rehabilitation, ${ }^{14}$ for floor effect in patients with severe brain damage, insensitivity of FIM to cognitive and psychosocial disability, insensitivity to change in BI, dependence of FIM on the health care system in the USA and many other. ${ }^{1}$ It has particularly been proposed that the various modifications of $\mathrm{BI}^{15-17}$ should be considered because of their psychometric qualities. However, some authors were not able to confirm its assumed improvement in sensitivity. ${ }^{18}$

A systematic analysis of ADL measures has been conducted by Law and Letts ${ }^{19}$ comparing the ADL measures available between 1960 and 1988 , among them the BI. Although the authors report poor internal consistency for BI their overall recommendation is positive. BI with some other measures shows the best reliability ( $\mathrm{see}$ also ${ }^{20}$ and validity (see also ${ }^{21}$ evidence, is short, and appears to discriminate among levels of ADL function. It has demonstrated evidence of the ability to predict function against other standards. It also belongs to the group of instruments with the best potential for responsive measuring change in ADL function. Its concurrent and predictive validity has been well established against extent of motor loss, ${ }^{22}$ prognosis, ${ }^{23}$ severity of stroke ${ }^{24}$ and other criteria.

The BI has predictive validity in terms of survival, ${ }^{25}$ functional recovery ${ }^{26}$ and other criteria. ${ }^{27}$ Walker and colleagues ${ }^{28}$ measured significant outcome differences with BI in the group of stroke survivors who were not admitted to hospital and did vs. did not receive occupational therapy. Alarcon and colleagues ${ }^{29}$ report that the only variable predictive of prolonged stay in hospital was a Barthel score of $<45$ on admission.

There are reports on reliability of BI indicating that test-retest reliability and inter-code reliability (22), inter-observer-reliability ${ }^{30}$ 
and inter-method reliability ${ }^{31}$ are satisfactory. However, it has also been indicated that BI scored upon a physicians' interview with a patient is not reliable compared to scoring based upon nurses' observation. ${ }^{32}$

As its sensitivity to small improvements in the rehabilitation process is relatively crude a modified index with a five-point scoring has been proposed. ${ }^{17}$ However, Wood-Dauphinee and co-workers ${ }^{27}$ report that $\mathrm{BI}$ was the most efficient measure and therefore required the fewest subjects to identify a significant outcome effect in stroke rehabilitation. The assessment by $\mathrm{BI}$ can also be reliably conducted by a telephone interview by health professional and lay-persons alike. ${ }^{33}$

The self-rating BI has been less extensively evaluated. ${ }^{34-36}$ Collin and colleagues ${ }^{20}$ found that Barthel Index as a self-report measure is highly reliable in a series of 25 consecutive admissions to an acute rehabilitation unit. However, they indicate that the most disagreement between self-report and observation was in the self-reports of cognitively impaired patients. Analysing the internal consistency they maintain that most of the disagreement was minor and was associated with more complex items such as transfers. Wade maintains that ADL indices should report actual performance in the relevant setting. ${ }^{37}$ It has been shown that the self-report BI is also reliable as a postal questionnaire as all items except 'toilet' and 'mobility' had at least moderate reliability by the kappa coefficient. ${ }^{36}$

The Functional Independence Measure (FIM) ${ }^{5,38}$ is a wellestablished and widely used measure of functional status. The first 13 items of the FIM represent a measure of motor function and the last 5 items represent a measure of cognitive function. It has been indicated that FIM item difficulties vary across impairment groups. ${ }^{39}$ The authors calculated item difficulties and report that 'feeding' and 'grooming' are the easiest motor items whereas 'stair climbing', 'tub/ shower transfers' and 'locomotion' are the most difficult ones. Among the cognitive items 'comprehension' and 'expression' are the easiest items, 'problem solving' is the most difficult.

Chau and colleagues ${ }^{40}$ studied inter rater agreement of the French version and report that all activities of self care, sphincter control, and of transfer, stair, expression and problems solving were rated with very good agreement (kappa coefficient higher than 0.80 ) and locomotion walk - wheelchair, comprehension, social interaction (kappa coefficient 0.7-0.8) and memory (kappa coefficient 0.63 ) with good agreement. They also found certain differences between the ratings by educators and by physio or occupational therapists.

There is a number of studies reporting using BI as well as FIM, comparing them and analysing their relationship. Van der Putten

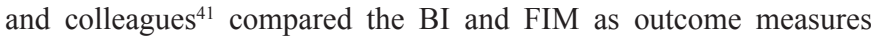
after inpatient rehabilitation and report that BI and FIM total, FIM motor and FIM cognitive scales were appropriate measures for the stroke patients, but that FIM cognitive scale has limited usefulness in progressive multiple sclerosis because of its notable ceiling effect. Nyein and McMichael ${ }^{42}$ and Turner-Stokes (1) demonstrated that BI can be derived from the FIM motor.

This article addresses the issue whether the ongoing measure with FIM and the self-report BI provides an adequate and satisfactory outcome measure in a community based rehabilitation centre and whether the patients improved in the course of rehabilitation

\section{Methods and Subjects}

This investigation uses data collected as part of the routine outcome measures in the Rehabilitation Centre. The patients are screened at the point of entry and prior to their discharge. The FIM data were collected by the nurses, the BI was administered by the occupational therapists. As the FIM assessment is possible to perform independently of the patients' abilities the FIM screening covers nearly $100 \%$ if the patients. BI (self-rating), on the other hand, relies on the language and cognitive abilities of the patient and was, therefore, not completed as often as FIM. Consequently, the data set contains only those patients for whom both of the screening measures are available $(\mathrm{N}=1033)$.

The table 1 describes the characteristics of the patients with FIM and self-report BI. The diagnoses are summarized in 6 groups. The median age is between 49 (patients after brain injury) and 80 years (geriatric multiorgan illness). The proportion of female patients lies between $41 \%$ (brain injury) and 63\% (geriatric multiorgan illness). The patients were referred mostly from an acute hospital (63\% of brain surgery patients; $80 \%$ of post surgery patients) with the exception of patients with rheumatological illness $(27 \%)$ and patients with other neurological illness (47\%) (Table 1).

Table I Patients' characteristics

\begin{tabular}{llllllllllllll}
\hline Diagnosis & $\mathbf{I}$ & & $\mathbf{2}$ & & $\mathbf{3}$ & & $\mathbf{4}$ & \multicolumn{3}{c}{$\mathbf{5}$} & $\mathbf{6}$ \\
\cline { 2 - 11 } & $\mathbf{N}$ & $\%$ & $\mathbf{N}$ & $\%$ & $\mathbf{N}$ & $\%$ & $\mathbf{N}$ & $\%$ & $\mathbf{N}$ & $\%$ & $\mathbf{N}$ & $\%$ \\
\hline Marital status & & & & & & & & & & & & \\
Single & 22 & 6.5 & 6 & 22.2 & 28 & 13.9 & 15 & 7.6 & 8 & 9.5 & 35 & 18.2 \\
Married & 214 & 63.5 & 14 & 51.9 & 97 & 48 & 128 & 65 & 40 & 47.6 & 111 & 57.8 \\
Separated & & & & & & & & & & & & \\
divorced & 24 & 7.1 & 1 & 3.7 & 19 & 9.4 & 15 & 7.6 & 2 & 2.4 & 15 & 7.8 \\
Widowed & 77 & 22.8 & 6 & 22.2 & 58 & 28.7 & 39 & 19.8 & 34 & 40.5 & 31 & 16.1 \\
Gender & & & & & & & & & & & & \\
Male & 153 & 45.4 & 11 & 40.7 & 109 & 54 & 118 & 59.9 & 53 & 63.1 & 114 & 59.4 \\
Female & 184 & 54.6 & 16 & 59.3 & 93 & 46 & 79 & 40.1 & 31 & 36.9 & 78 & 40.6 \\
Referred from: & & & & & & & & & & & & \\
Home & 91 & 27 & 6 & 22.2 & 31 & 15.3 & 134 & 68 & 21 & 25 & 90 & 46.9 \\
\hline
\end{tabular}




\begin{tabular}{|c|c|c|c|c|c|c|c|c|c|c|c|c|}
\hline \multirow[t]{2}{*}{ Diagnosis } & \multicolumn{2}{|l|}{$\mathbf{I}$} & \multicolumn{2}{|l|}{2} & \multicolumn{2}{|l|}{3} & \multicolumn{2}{|l|}{4} & \multicolumn{2}{|l|}{5} & \multicolumn{2}{|l|}{6} \\
\hline & $\mathbf{N}$ & $\%$ & $\mathbf{N}$ & $\%$ & $\mathbf{N}$ & $\%$ & $\mathbf{N}$ & $\%$ & $\mathbf{N}$ & $\%$ & $\mathbf{N}$ & $\%$ \\
\hline Acute hospital Rehab. & 233 & 6901 & 17 & 63 & 162 & 80.2 & 55 & 27.9 & 58 & 69 & 89 & 46.4 \\
\hline Clinic Care & 0 & 0 & 0 & 0 & 2 & 1 & 1 & 5 & 0 & 0 & 3 & 1.6 \\
\hline institution & 6 & 1.8 & 2 & 7.4 & 2 & 1 & 5 & 2.5 & 3 & 3.6 & 6 & 3.1 \\
\hline Other & 5 & 1.5 & 2 & 7.4 & 5 & 2.5 & 2 & 1 & 2 & 2.4 & 4 & 2.1 \\
\hline \multicolumn{13}{|l|}{ Referred to: } \\
\hline Home & 271 & 80.4 & 25 & 92.6 & 155 & 76.7 & 171 & 86.8 & 63 & 75 & 155 & 80.7 \\
\hline $\begin{array}{l}\text { Acute hospital } \\
\text { Rehab. }\end{array}$ & 4 & 1.2 & 0 & 0 & 8 & 4 & 5 & 2.5 & 4 & 4.8 & 5 & 2.6 \\
\hline Clinic Care & 3 & 0.9 & 0 & 0 & 3 & 1.5 & 2 & 1 & 2 & 2.4 & 4 & 2.1 \\
\hline institution & 37 & 11 & 2 & 7.4 & 22 & 10.9 & 11 & 5.6 & 11 & 3.1 & 21 & 10.9 \\
\hline Other & 32 & 6.5 & 0 & 0 & 14 & 6.9 & 8 & 4.1 & 4 & 4.8 & 7 & 3.6 \\
\hline \multicolumn{13}{|l|}{ Year of admission: } \\
\hline 1995 & 91 & 27 & 3 & 11.1 & 34 & 16.8 & 37 & 18.8 & 25 & 29.8 & 33 & 17.2 \\
\hline 1996 & 60 & 17.8 & 6 & 22.2 & 35 & 17.3 & 37 & 18.8 & 15 & 17.9 & 35 & 18.2 \\
\hline 1997 & 52 & 15.4 & 7 & 25.9 & 38 & 18.8 & 41 & 20.8 & 16 & 19 & 44 & 22.9 \\
\hline 1998 & 61 & 18.1 & 5 & 18.5 & 44 & 21.8 & 38 & 19.3 & 16 & 19 & 37 & 19.3 \\
\hline 1999 & 56 & 16.6 & 5 & 18.5 & 32 & 15.8 & 31 & 15.7 & 7 & 8.3 & 33 & 17.2 \\
\hline 2000 & 17 & 5 & 1 & 9 & 19 & 9.4 & 13 & 6.6 & $\underline{5}$ & 6 & 10 & 5.2 \\
\hline \multicolumn{13}{|l|}{ Age } \\
\hline Mean & 68.9 & & 50 & & 72.5 & & 58.7 & & 79.3 & & 61.6 & \\
\hline Median & 71 & & 49 & & 75 & & 55 & & 80 & & 65 & \\
\hline stddev & 13.28 & & 21.13 & & 12.566 & & 18.993 & & 9.081 & & $16.06 \mid$ & \\
\hline
\end{tabular}

\section{Diagnoses}

(I)Vascular brain damage $\quad(\mathrm{N}=337)$

(2)Brain injury $\quad(\mathrm{N}=27)$

(3)Post surgery

$(\mathrm{N}=27)$
$(\mathrm{N}=202)$

(4)Rheumatologic illness $\quad(\mathrm{N}=197)$

(5) Geriatric multiorgan illness $(N=84)$

(6) other neurological ilness $\quad(N=192)$

$(\mathrm{N}=1017)$

\section{Instruments}

Barthel Index is a scale of activities of daily living that includes 10 fundamental items from 2- to 4-level scales and is one of the standard measuring devices of functional disabilities or resources. We used the modified version by Granger and colleagues ${ }^{16,22,43}$ to measure each patient's ADL performance on admission and on discharge. Fifteen items related to self-care ability, continence and mobility are scored by determining whether the patient can perform them independently, with assistance or supervision, or not at all. The scores for each item are summed and the total ranges from 0 (complete dependence) to 100 (independence in terms of ADL). The BI can be divided to measure the discrete functions of self-care ( $0-53$ points) or mobility (0-47 points). The Barthel Index was originally developed as a means of assessing the level of independence in patients with neuromuscular or musculoskeletal disorders.

The Functional Independence Measure (FIM) is an 18 item observational sheet and interview schedule. Each of the patient's activities (self care, sphincter management, transfer, locomotion, communication and social cognition) is rated between 1 (totally dependent) and 7 (fully independent). The FIM was filled out for every patient treated at the Rehabilitation Centre of the Medical Clinic, Buerger Hospital in Solothurn during 1995-2000. Additional personal and socio demographic data were also collected. This evaluation contains data of the observations made on admission and on discharge of the patients.

\section{The setting}

The Rehabilitation Centre is a 25 bed in patient hospital adjoined to an acute hospital in a small town in Switzerland. It cares for a broad range of patients although recently the rehabilitation of geriatric multi organ illnesses was outsourced to another centre. The rehabilitation team is active in implementing and optimising treatment informed by M. Johnstone's teaching and is in process of restructuring the rehabilitation organization according to the Total Quality Management principles and it reached good benchmarking results in rehabilitation outcome ${ }^{44}$ and in time spent with patients. ${ }^{45}$ 


\section{Results}

\section{Floor and ceiling effects of FIM and BI}

As there were several indications and complaints about the floor and ceiling effects of the used instruments a simple floor and ceiling effect check was performed. The Table 2 summarizes the amount of patients in \% who assessed or were assessed with the lowest degree of independence on the admission measure. There are some variation in the items and in the type of diagnosis the patients received. The items 'bathing' and 'washing' contain a large number of patients with the lowest rating thus indicating a possible floor effect. The group of post surgery patients contains the highest number of patients with the lowest rating in the highest number of items suggesting that in this group of patients some functions may not be sufficiently differentiated in the lower rating. 'Drinking' seems to posses the highest ceiling effect in BI. The patients with rheumatological illness reached the highest number of the patients with the highest rating in the most items. Consequently, an improvement in these patients could not be monitored by this measure. Using the total sum of all items we found less floor effects (between 0 and $7.4 \%$ of patients of a diagnosis group) and less ceiling effect (between 8 and $47 \%$ ). The highest total sum on discharge as a measure of a ceiling effect suggests between 17 and $70 \%$ of patients who could have not scored higher because of the limitation of the scale. The BI self care scale showed a lower floor effect and higher ceiling effect that the mobility scale (Table 2).

Table 2 Floor and ceilling effect in mod. Barthel index (self assessment) Floor effect (the amount of patient with the lowest level on admission in \%)

\begin{tabular}{|c|c|c|c|c|c|c|}
\hline Diagnosis & I & 2 & 3 & 4 & 5 & 6 \\
\hline Drinking & 5 & 7.4 & I & 0.5 & 2.4 & 7.8 \\
\hline Eating & 22 & 18.5 & 6.9 & 4 & 14.3 & 16.1 \\
\hline Upper body dressing & 5.3 & 7.4 & I & 0 & 0 & 4.7 \\
\hline Lower body dressing & 14.5 & 7.4 & 9.9 & 2 & 7.1 & I5.I \\
\hline $\begin{array}{l}\text { Application of mech. } \\
\text { devices }\end{array}$ & 8 & 3.7 & 16.3 & $\mathrm{I}$ & 9.5 & 5.2 \\
\hline Personal hygiene & 35.9 & 18.5 & 32.7 & 15.7 & 33.3 & 35.4 \\
\hline Washing & 43 & 33.3 & 53 & 22.8 & 45.2 & 43.2 \\
\hline Bladder control & 8.9 & II.I & 6.4 & 2 & 8.3 & 14.1 \\
\hline Bowel control & 4.5 & II.I & 3 & 1 & 1.2 & 6.3 \\
\hline Getting in/out of bed & 5.3 & 7.4 & 4 & 1 & 4.8 & 8.9 \\
\hline Toilet & 7.4 & 7.4 & 6.9 & 13.2 & 4.8 & 7.8 \\
\hline Bathing & 67.7 & 44.4 & 77.2 & 33.5 & 81 & 58.9 \\
\hline Walking & 28.2 & 14.8 & 32.2 & 9.6 & 26.2 & 27.1 \\
\hline Stairs & 30.6 & 18.5 & 52 & 15.7 & 31 & 30.7 \\
\hline
\end{tabular}

Ceilling effect (the amount of patient with the highest level on admission in $\%)$

\begin{tabular}{lllllll}
\hline Diagnosis & I & $\mathbf{2}$ & $\mathbf{3}$ & $\mathbf{4}$ & $\mathbf{5}$ & $\mathbf{6}$ \\
\hline Drinking & 95 & 92.6 & 99 & 99.5 & 97.6 & 92.2 \\
Eating & 78 & 81.5 & 93.1 & 95.9 & 85.7 & 83.9 \\
Upper body dressing & 61.1 & 74.1 & 88.1 & 91.9 & 69 & 72.4 \\
Lower body dressing & 50.1 & 66.7 & 34.7 & 66.5 & 42.9 & 50
\end{tabular}

$\begin{array}{lllllll}\begin{array}{l}\text { Application of mech. } \\ \text { devices }\end{array} & 92 & 96.3 & 83.7 & 99 & 90.5 & 94.8 \\ \text { Personal hygiene } & 64.1 & 81.5 & 67.3 & 84.3 & 66.7 & 64.6 \\ \text { Washing } & 57 & 66.7 & 47 & 77.2 & 54.8 & 56.8 \\ \text { Bladder control } & 80.1 & 77.8 & 83.7 & 91.4 & 71.4 & 67.7 \\ \text { Bowel control } & 88.7 & 85.2 & 92.1 & 95.4 & 92.9 & 80.2 \\ \text { Getting in/out of bed } & 58.5 & 74.1 & 51.5 & 82.7 & 56 & 57.8 \\ \text { Toilet } & 55.8 & 77.8 & 48.5 & 86.8 & 58.3 & 62 \\ \text { Bathing } & 32.3 & 55.6 & 22.8 & 66.5 & 19 & 41.1 \\ \text { Walking } & 45.1 & 63 & 41.6 & 79.7 & 47.6 & 51 \\ \text { Stairs } & 39.5 & 70.4 & 20.8 & 67 & 36.9 & 39.1\end{array}$

\section{Diagnoses}

(I)Vascular brain damage $\quad(\mathrm{N}=337)$

(2)Brain injury $\quad(\mathrm{N}=27)$

(3)Post surgery $\quad(\mathrm{N}=202)$

(4)Rheumatologic illness $\quad(\mathrm{N}=197)$

(5) Geriatric multiorgan illness $(\mathrm{N}=84)$

(6)Other neurological ilness $\quad(\mathrm{N}=192)$

Although there are more possibilities to differentiate in FIM because of the 1-7 value scales the floor and ceiling effects are also noticeable (Table 3). The items differ but the highest number of patients with the lowest assessment can be see in regard to "climbing stairs' in all diagnosis groups (17-59\%). The ceiling effect is indicated by a high number of patients reaching the highest rating on admission for 'bowel management ' and for 'eating'. However, the amount of patients reaching the highest rating in all 18 items on admission and thus not being able to respond with improvement is relatively low (between 0.3 to $3.7 \%$ with the exception of patients with rheumatological illness (14\%) (Table 3).

Table 3 Floor and ceilling effect in FIM Floor effect (the amount of patients with the lowest FIM level on admission in \%)

\begin{tabular}{lllllll}
\hline Diagnosis & $\mathbf{I}$ & $\mathbf{2}$ & $\mathbf{3}$ & $\mathbf{4}$ & $\mathbf{5}$ & $\mathbf{6}$ \\
\hline A. Eating & 2.7 & 7.4 & 0.5 & 0 & 1.2 & 4.2 \\
B. Grooming & 4.7 & 7.4 & 1 & 0.5 & 3 & 8.3 \\
$\begin{array}{l}\text { C. Bathing } \\
\text { D. Upper body }\end{array}$ & 18.4 & 14.8 & 12.4 & 5 & 11.9 & 19.8 \\
dressing & 8 & 11.1 & 3.5 & 2 & 4.8 & 10.4 \\
$\begin{array}{l}\text { E. Lower body } \\
\text { dressing }\end{array}$ & 33.8 & 18.5 & 41.6 & 10.7 & 34.5 & 37 \\
$\begin{array}{l}\text { F. Toileting } \\
\text { G. Bladder }\end{array}$ & 31.5 & 18.5 & 26.7 & 8.6 & 32.1 & 34.4 \\
management & 14.8 & 14.8 & 7.4 & 3.6 & 14.3 & 18.8 \\
H. Bowel management & 7.1 & 14.8 & 1.5 & 1 & 4.8 & 10.9 \\
I. Bed/chair/WC & 10.1 & 11.1 & 4 & 2 & 8.3 & 13 \\
J.Toilet & 15.4 & 14.8 & 6.4 & 2 & 9.5 & 15.6 \\
K.Tub/shower & 24.3 & 18.5 & 21.3 & 5.1 & 22.6 & 20.8 \\
L.Walk/WC & 18.4 & 14.8 & 14.8 & 3 & 13.1 & 10.9 \\
\hline M. Stairs & 39.5 & 29.6 & 58.9 & 16.8 & 40.5 & 40.6 \\
N. Comprehension & 1.2 & 7.4 & 0.5 & 0 & 1.2 & 1 \\
O. Expression & 5 & 14.8 & 0.5 & 0 & 1.2 & 2.6 \\
\hline
\end{tabular}


Table Continued

\begin{tabular}{lllllll}
\hline Diagnosis & I & $\mathbf{2}$ & $\mathbf{3}$ & $\mathbf{4}$ & $\mathbf{5}$ & $\mathbf{6}$ \\
\hline P. Social interaction & $\mathrm{I} .5$ & $\mathrm{II} . \mathrm{I}$ & $\mathrm{I} .5$ & 0 & 2.4 & 1.6 \\
Q. Problem solving & $\mathrm{II}$ & $\mathrm{II} . \mathrm{I}$ & 2.5 & 0.5 & 9.5 & 9.4 \\
R. Memory & 3.3 & $\mathrm{II} . \mathrm{I}$ & $\underline{1}$ & $\underline{0}$ & 2.4 & 3.6 \\
\hline
\end{tabular}

Ceilling effect:(the amount of patients with the highest level on admission in \%)

\begin{tabular}{|c|c|c|c|c|c|c|c|}
\hline Diagnosis & I & 2 & 3 & 4 & 5 & 6 & All \\
\hline A. Eating & 23.4 & 44.4 & $\underline{62.9}$ & $\underline{80.7}$ & $\underline{50}$ & $\underline{47.9}$ & \\
\hline B. Grooming & 21.1 & 37 & 28.2 & 66.5 & 22.6 & 29.2 & \\
\hline C. Bathing & 8 & 18.5 & 8.4 & 40.6 & 4.8 & 12 & \\
\hline $\begin{array}{l}\text { D. Upper body } \\
\text { dressing }\end{array}$ & 21.1 & 22.2 & 32.7 & 62.9 & 21.4 & 28.6 & \\
\hline $\begin{array}{l}\text { E. Lower body } \\
\text { dressing }\end{array}$ & 16.6 & 18.5 & 11.9 & 43.7 & 13.1 & 21.9 & \\
\hline F.Toileting & 21.1 & 33.3 & 24.8 & 67 & 17.9 & 27.1 & \\
\hline $\begin{array}{l}\text { G. Bladder } \\
\text { management }\end{array}$ & 43.3 & 66.7 & 45.5 & 76.1 & 33.3 & 40.6 & \\
\hline $\begin{array}{l}\text { H. Bowel } \\
\text { management }\end{array}$ & $\underline{49.3}$ & $\underline{74.1}$ & 46.5 & 76.6 & 39.3 & 44.3 & \\
\hline I. Bed/chair/WC & 22.8 & 29.6 & 12.4 & 54.8 & 14.3 & 22.9 & \\
\hline J.Toilet & 28.2 & 37 & 20.3 & 59.4 & 15.5 & 24 & \\
\hline K.Tub/shower & 15.7 & 18.5 & 8.4 & 44.2 & 7.1 & 15.1 & \\
\hline L.Walk/WC & 16.3 & 18.5 & 6.9 & 39.6 & 4.8 & 18.2 & \\
\hline M. Stairs & 8.3 & 14.8 & 3.5 & 27.4 & 3.6 & 13 & \\
\hline $\begin{array}{l}\text { N. } \\
\text { Comprehension }\end{array}$ & 35.6 & 33.3 & 50 & 62.4 & 44 & 45.3 & \\
\hline O. Expression & 29.1 & 29.6 & 54 & 65.5 & 46.4 & 45.8 & \\
\hline $\begin{array}{l}\text { P. Social } \\
\text { interaction }\end{array}$ & 39.5 & 29.6 & 56.9 & 68.5 & 38.1 & 44.8 & \\
\hline $\begin{array}{l}\text { Q. Problem } \\
\text { solving }\end{array}$ & 13.6 & 18.5 & 29.2 & 52.8 & 17.9 & 20.8 & \\
\hline R. Memory & 23.4 & 18.5 & 36.6 & 68.5 & 23.8 & 38.5 & \\
\hline Total & 0.3 & 3.7 & 1.5 & 14.5 & 1.2 & 2.1 & 3.5 \\
\hline
\end{tabular}

Diagnoses:

(I) Vascular brain damage $(\mathrm{N}=337)$

(2) Brain injury $(\mathrm{N}=27)$

(3) Post surgery ( $\mathrm{N}=202)$

(4) Rheumatologic illness $(\mathrm{N}=197)$

(6) other neurological ilness ( $N=192)$

This assessment of floor and ceiling effects is very crude as there are many items in BI with just a two value scale as compared to 7 point scale of the FIM.

However, it indicates substantial differences between items (eating, understanding: between 0 and $7.4 \%$ of patients indicate the lowest value; stairs climbing: $17-59 \%$ of patients indicate the lowest value), between diagnosis groups (rheumatologic illness: $17 \%$ and post surgery patients: $59 \%$ indicate the lowest value in one of the functions) and between measures (BI: $81 \%$ of the patients of one diagnosis group indicate in regard to one function the lowest value compared to FIM with $59 \%$ ).

Similarly, the ceiling effect is also stronger in BI $(99.5 \%$ of the patients of one diagnosis group report the highest value in regard to
(5) Geriatric multiorgan illness $(\mathrm{N}=84)$

one function) than in FIM (80.7\%).

\section{Factor structure of FIM}

The widespread practice of adding all values of the 18 FIM items into an overall score has been occasionally questioned. Although the FIM items correlate highly among themselves and the reliability coefficient alpha is very high, the issue of the linearity, interchangeability, equal distances etc. is still open. Recently, Ravaud and colleagues ${ }^{46}$ warned of using the FIM total score without precaution because of the multidimensionality of the FIM items. They recommend using various subscores. Consequently, before using FIM scale it is advisable to analyse the factorial structure of FIM as gained with the given sample.

We performed a factor analysis with the patients of each diagnostic group separately for the admission and discharge data. The admission FIM total data in patients with vascular brain damage, with brain injury and with other neurological illness were presented as being two dimensional separating clearly between FIM motor and FIM cognitive and accounting for $73.6 \%, 87.5 \%$ and $72.8 \%$ (Tables 4 , 5 ). We obtained a 3 dimensional solution in post surgery patients (accounting for 68.7\%), in patients with rheumatology illness (accounting for $78 \%$ ) and in patients with geriatric multiorgan illness (accounting for $70.1 \%$ ). The third factor comprises either of 'eating', 'grooming' and 'upper body dressing' as in post surgery patients or of 'comprehension' and 'expression' as in the other two patient groups (Table 4) (table 5)

Table 4 Factor analysis FIM all items (admission)

\begin{tabular}{|c|c|c|c|}
\hline Factor & Eigenvalue & Pct of var & Cum pct \\
\hline I & \multicolumn{2}{|c|}{ Vascular brain damage } & $(\mathrm{N}=337)$ \\
\hline I & 11.006 & 61.1 & 61.1 \\
\hline 2 & 2.245 & 12.5 & 73.6 \\
\hline ॥ & \multicolumn{2}{|l|}{ Brain injury } & $(N=27)$ \\
\hline I & 13.559 & 75.3 & 75.3 \\
\hline 2 & 2.197 & 12.2 & 87.5 \\
\hline III & \multicolumn{2}{|c|}{ Post surgery } & $(\mathrm{N}=202)$ \\
\hline I & 8.906 & 49.5 & 49.5 \\
\hline 2 & 2.219 & 12.3 & 61.8 \\
\hline 3 & 1.239 & 6.9 & 68.7 \\
\hline IV & \multicolumn{2}{|c|}{ Rheumatologic illness } & $(N=197)$ \\
\hline I & 10.377 & 57.7 & 57.7 \\
\hline 2 & 2.636 & 14.6 & 72.3 \\
\hline 3 & 1.019 & 5.7 & 78 \\
\hline v & \multicolumn{2}{|c|}{ Geriatric multiorgan illness } & $(N=84)$ \\
\hline I & 8.446 & 46.9 & 46.9 \\
\hline Factor & Eigenvalue & Pct of var & Cum pct \\
\hline 2 & 2.627 & 14.6 & 61.5 \\
\hline 3 & 1.543 & 8.6 & 70.1 \\
\hline VI & \multicolumn{2}{|c|}{ other neurological ilness } & $(\mathrm{N}=192)$ \\
\hline I & 10.055 & 55.9 & 55.9 \\
\hline 2 & 3.046 & 16.9 & 72.8 \\
\hline
\end{tabular}

Citation:Valach L, Selz B, Hofer K, et al.A six-year audit in a rehabilitation centre with mod barthel index and FIM as outcome measures. Int Phys Med Rehab J. 2018;3(4):342-358. DOI: I0.15406/ipmrj.2018.03.00I28 
Table 5 Factor analysis of FIM items on admission in each diagnosis Rotated factor matrix (Diagnosis, factor):

\begin{tabular}{|c|c|c|c|c|c|c|c|}
\hline & I.I & 1.2 & 2.1 & 2.2 & 3.1 & 3.2 & 3.3 \\
\hline Eating & 0.62 & 0.3 & 0.55 & 0.54 & 0.13 & 0.14 & 0.87 \\
\hline Grooming & 0.76 & 0.39 & 0.73 & 0.58 & 0.34 & 0.2 & 0.69 \\
\hline Bathing & 0.85 & 0.29 & 0.85 & 0.34 & 0.65 & 0.15 & 0.52 \\
\hline $\begin{array}{l}\text { Upper body } \\
\text { dressing }\end{array}$ & 0.84 & 0.3 & 0.81 & 0.54 & 0.44 & 0.22 & 0.72 \\
\hline $\begin{array}{l}\text { Lower body } \\
\text { dressing }\end{array}$ & 0.89 & 0.19 & 0.89 & 0.37 & 0.73 & 0.22 & 0.36 \\
\hline Toileting & 0.9 & 0.25 & 0.88 & 0.31 & 0.73 & 0.27 & 0.37 \\
\hline $\begin{array}{l}\text { Bladder } \\
\text { management }\end{array}$ & 0.63 & 0.5 & 0.75 & 0.56 & 0.46 & 0.39 & 0.29 \\
\hline $\begin{array}{l}\text { Bowel } \\
\text { management }\end{array}$ & 0.59 & 0.52 & 0.76 & 0.56 & 0.44 & 0.38 & 0.22 \\
\hline Bed/chair/WC & 0.9 & 0.2 & 0.9 & 0.35 & 0.83 & 0.22 & 0.24 \\
\hline Toilet & 0.92 & 0.18 & 0.91 & 0.34 & 0.84 & 0.24 & 0.26 \\
\hline Tub/shower & 0.9 & 0.18 & 0.93 & 0.25 & 0.81 & 0.15 & 0.34 \\
\hline Walk/WC & 0.84 & 0.27 & 0.83 & 0.45 & 0.76 & 0.15 & 0.16 \\
\hline Stairs & 0.85 & 0.18 & 0.91 & 0.04 & 0.73 & 0.09 & -0.05 \\
\hline Comprehension & 0.1 & 0.83 & 0.39 & 0.88 & 0.03 & 0.83 & 0.2 \\
\hline Expression & 0.12 & 0.81 & 0.26 & 0.89 & 0.09 & 0.82 & 0.27 \\
\hline $\begin{array}{l}\text { Social } \\
\text { interaction }\end{array}$ & 0.3 & 0.72 & 0.2 & 0.92 & 0.19 & 0.79 & 0.08 \\
\hline Problem solving & 0.37 & 0.78 & 0.39 & 0.85 & 0.31 & 0.83 & 0.11 \\
\hline Memory & 0.24 & 0.79 & 0.25 & 0.91 & 0.35 & 0.73 & 0 \\
\hline
\end{tabular}

\begin{tabular}{|c|c|c|c|c|c|c|c|c|}
\hline & 4.1 & 4.2 & 4.3 & 5.1 & 5.2 & 5.3 & 6.1 & 6.2 \\
\hline Eating & 0.56 & 0.18 & 0.38 & 0.49 & 0.32 & 0.16 & 0.64 & 0.27 \\
\hline Grooming & 0.81 & 0.15 & 0.2 & 0.65 & 0.23 & -0.06 & 0.76 & 0.3 \\
\hline Bathing & 0.89 & 0.24 & 0.07 & 0.82 & 0.22 & -0.08 & 0.87 & 0.2 \\
\hline $\begin{array}{l}\text { Upper body } \\
\text { dressing }\end{array}$ & 0.87 & -0.02 & 0.19 & 0.79 & 0.33 & 0.08 & 0.84 & 0.27 \\
\hline $\begin{array}{l}\text { Lower body } \\
\text { dressing }\end{array}$ & 0.85 & 0.19 & 0.05 & 0.81 & 0.26 & 0.02 & 0.87 & 0.15 \\
\hline Toileting & 0.88 & 0.24 & 0.04 & 0.79 & 0.29 & 0.03 & 0.87 & 0.19 \\
\hline $\begin{array}{l}\text { Bladder } \\
\text { management }\end{array}$ & 0.8 & 0.26 & 0.03 & 0.38 & 0.65 & -0.07 & 0.75 & 0.27 \\
\hline $\begin{array}{l}\text { Bowel } \\
\text { management }\end{array}$ & 0.73 & 0.26 & 0.15 & 0.45 & 0.58 & -0.04 & 0.75 & 0.23 \\
\hline Bed/chair/WC & 0.91 & 0.1 & 0.07 & 0.89 & 0.08 & 0.09 & 0.91 & 0.1 \\
\hline Toilet & 0.89 & 0.2 & 0.04 & 0.87 & 0.24 & -0.03 & 0.92 & 0.07 \\
\hline Tub/shower & 0.89 & 0.18 & -0.06 & 0.82 & 0.33 & -0.11 & 0.9 & 0.07 \\
\hline Walk/WC & 0.84 & 0.19 & 0.1 & 0.79 & -0.01 & 0.14 & 0.8 & 0.2 \\
\hline Stairs & 0.79 & 0.25 & 0.05 & 0.8 & -0.07 & 0.15 & 0.78 & -0.02 \\
\hline Comprehension & 0.07 & 0.28 & 0.92 & 0 & 0.18 & 0.94 & 0.09 & 0.87 \\
\hline
\end{tabular}


Table Continued

\begin{tabular}{|c|c|c|c|c|c|c|c|c|}
\hline & I.I & 1.2 & 2.1 & 2.2 & 3.1 & 3.2 & 3.3 & \\
\hline Expression & 0.05 & 0.28 & 0.92 & 0.11 & 0.18 & 0.93 & 0.17 & 0.87 \\
\hline $\begin{array}{l}\text { Social } \\
\text { interaction }\end{array}$ & 0.2 & 0.86 & 0.24 & 0.15 & 0.82 & 0.09 & 0.11 & 0.8 \\
\hline Problem solving & 0.28 & 0.82 & 0.29 & 0.11 & 0.84 & 0.27 & 0.25 & 0.84 \\
\hline Memory & 0.26 & 0.82 & 0.19 & 0.07 & 0.8 & 0.26 & 0.2 & 0.87 \\
\hline
\end{tabular}

Diagnoses

(I) Vascular brain damage $(\mathrm{N}=337)$

(2) Brain injury $(\mathrm{N}=27)$

(3) Post surgery $(\mathrm{N}=202)$

(4) Rheumatologic illness ( $N=197)$

(5) Geriatric multiorgan illness $(\mathrm{N}=84)$

(6) other neurological ilness $(\mathrm{N}=192)$

Factor analysing the discharge FIM data we obtained two-factorial solution only in vascular brain diseases patients (accounting for $74.2 \%$ ) and in patients with other neurological illness (accounting for 74\%) (Table 6). The FIM data of patients with traumatic brain injury, rheumatological illness and geriatric multiorgan illness indicated three dimensions. The third dimension comprises of 'bladder management', 'bowel management' and 'transfer to toilet' in traumatic brain injury patients and of 'comprehension' and 'expression' in the other two groups of patients (Table 7).

Table 6 Factor analysis (principal-components analysis $(\mathrm{pc})$, varimax rotation, kaiser normalization) FIM all items (discharge)

\begin{tabular}{llll}
\hline Factor & Eigenvalue & \multicolumn{1}{l}{ Pct of var } & Cum pct \\
\hline I & Vascular brain damage & $(\mathrm{N}=337)$ \\
I & II.31694 & 62.9 & 62.9 \\
2 & 2.04397 & 11.4 & 74.2 \\
II & Brain injury & & $(\mathrm{N}=27)$ \\
I & II.69436 & 65 & 65 \\
2 & 2.34747 & 13 & 78 \\
3 & I.10984 & 6.2 & 84.2
\end{tabular}

\begin{tabular}{llll} 
III & \multicolumn{1}{l}{ Post surgery } & & $(\mathrm{N}=202)$ \\
I & 9.09305 & 50.5 & 50.5 \\
2 & 2.18527 & 12.1 & 62.7 \\
3 & 1.12119 & 6.2 & 68.9 \\
4 & 1.0003 & 5.6 & 74.4 \\
IV & Rheumatologic illness & $(\mathrm{N}=197)$ \\
I & 10.5052 & 58.4 & 58.4 \\
2 & 2.60032 & 14.4 & 72.8 \\
3 & 1.04344 & 5.8 & 78.6 \\
V & Geriatric multiorgan illness & $(\mathrm{N}=84)$ \\
I & 8.67759 & 48.2 & 48.2 \\
2 & 2.50119 & 13.9 & 62.1 \\
3 & 1.77356 & 9.9 & 72 \\
VI & other neurological ilness & $(\mathrm{N}=192)$ \\
I & 10.28102 & 57.1 & 57.1 \\
2 & 3.03811 & 16.9 & 74 \\
\hline
\end{tabular}

Table 7: Factor analysis (principal-components analysis (pc), varimax rotation, kaiser normalization)

FIM all items (discharge) Rotated factor matrix: (Diagnosis; factor)

\begin{tabular}{|c|c|c|c|c|c|c|c|c|c|}
\hline & I.I & 1.2 & 2.1 & 2.2 & 2.3 & 3.1 & 3.2 & 3.3 & 3.4 \\
\hline Eating & 0.69 & 0.24 & 0.81 & 0.25 & 0.19 & 0.19 & 0.26 & 0.75 & 0.06 \\
\hline Grooming & 0.79 & 0.37 & 0.74 & 0.61 & 0.05 & 0.27 & 0.23 & 0.78 & 0.18 \\
\hline Bathing & 0.87 & 0.32 & 0.8 & 0.28 & 0.26 & 0.54 & 0.13 & 0.53 & 0.3 \\
\hline $\begin{array}{l}\text { Upper body } \\
\text { dressing }\end{array}$ & 0.85 & 0.32 & 0.74 & 0.5 & 0.3 & 0.39 & 0.25 & 0.74 & 0.17 \\
\hline $\begin{array}{l}\text { Lower body } \\
\text { dressing }\end{array}$ & 0.87 & 0.31 & 0.77 & 0.42 & 0.29 & 0.61 & 0.12 & 0.57 & 0.14 \\
\hline Toiletting & 0.88 & 0.31 & 0.65 & 0.54 & 0.44 & 0.68 & 0.21 & 0.46 & 0.29 \\
\hline $\begin{array}{l}\text { Bladder } \\
\text { management }\end{array}$ & 0.67 & 0.38 & 0.31 & 0.29 & 0.86 & 0.3 & 0.29 & 0.14 & 0.8 \\
\hline $\begin{array}{l}\text { Bowel } \\
\text { management }\end{array}$ & 0.65 & 0.34 & 0.29 & 0.27 & 0.88 & 0.23 & 0.22 & 0.23 & 0.83 \\
\hline Bed/chair/WC & 0.91 & 0.16 & 0.67 & 0.29 & 0.63 & 0.82 & 0.19 & 0.27 & 0.11 \\
\hline Toilet & 0.92 & 0.17 & 0.61 & 0.23 & 0.72 & 0.81 & 0.11 & 0.25 & 0.33 \\
\hline Tub/shower & 0.89 & 0.24 & 0.63 & 0.27 & 0.48 & 0.78 & 0.18 & 0.17 & 0.34 \\
\hline
\end{tabular}




\begin{tabular}{|c|c|c|c|c|c|c|c|c|c|}
\hline & I.I & 1.2 & 2.1 & 2.2 & 2.3 & 3.1 & 3.2 & 3.3 & 3.4 \\
\hline Walk/WC & 0.85 & 0.19 & 0.72 & 0.12 & 0.53 & 0.71 & 0.21 & 0.21 & -0.02 \\
\hline Stairs & 0.86 & 0.21 & 0.82 & -5 & 0.3 & 0.78 & 0.13 & 0.13 & 0.11 \\
\hline Comprehension & 0.14 & 0.82 & 0.26 & 0.86 & 0.26 & 0.05 & 0.78 & 0.18 & 0.17 \\
\hline Expression & 0.18 & 0.8 & 0.25 & 0.8 & 0.12 & 0.08 & 0.74 & 0.32 & 0.24 \\
\hline $\begin{array}{l}\text { Social } \\
\text { interaction }\end{array}$ & 0.3 & 0.69 & 0.1 & 0.91 & 0.12 & 0.11 & 0.78 & 0.19 & 0.09 \\
\hline Problem solving & 0.37 & 0.79 & 0.25 & 0.82 & 0.27 & 0.26 & 0.82 & 0.17 & 0.18 \\
\hline \multirow[t]{2}{*}{ Memory } & 0.26 & 0.8 & 0.17 & 0.82 & 0.23 & 0.33 & 0.81 & 0.03 & 0.04 \\
\hline & & 4.1 & 4.2 & 4.3 & 5.1 & 5.2 & 5.3 & 6.1 & 6.2 \\
\hline Eating & & .49 & 0.47 & -0.01 & 0.47 & 0.34 & 0.27 & 0.6 & 0.3 \\
\hline Grooming & & .86 & 0.25 & 0.09 & 0.71 & 0.04 & 0.28 & 0.78 & 0.35 \\
\hline Bathing & & 0.83 & 0.36 & -0.01 & 0.82 & 0.31 & 0.01 & 0.85 & 0.2 \\
\hline Upper body dressin & & 0.91 & 0.18 & 0.06 & 0.81 & 0.08 & 0.25 & 0.86 & 0.3 \\
\hline Lower body dressir & & 0.81 & 0.36 & -0.01 & 0.81 & 0.19 & 0.04 & 0.88 & 0.15 \\
\hline Toiletting & & 0.83 & 0.33 & -0.03 & 0.82 & 0.33 & 0 & 0.88 & 0.21 \\
\hline Bladder managemen & & 0.89 & 0.09 & 0.07 & 0.63 & 0.45 & -0.13 & 0.79 & 0.21 \\
\hline Bowel management & & 0.83 & 0.07 & 0.03 & 0.68 & 0.43 & -0.1 & 0.82 & 0.14 \\
\hline Bed/chair/WC & & 0.88 & 0.17 & 0.11 & 0.91 & 0.06 & -0.02 & 0.92 & 0.1 \\
\hline Toilet & & 0.91 & 0.13 & 0.17 & 0.9 & 0.09 & 0.02 & 0.93 & 0.09 \\
\hline Tub/shower & & 0.88 & 0.2 & 0.02 & 0.79 & 0.26 & 0.01 & 0.92 & 0.08 \\
\hline Walk/WC & & 0.8 & 0.16 & 0.13 & 0.78 & -0.06 & 0.08 & 0.77 & 0.2 \\
\hline Stairs & & 0.79 & 0.26 & 0.02 & 0.8 & -0.06 & 0.07 & 0.8 & 0.02 \\
\hline Comprehension & & 0.06 & 0.27 & 0.93 & 0.03 & 0.08 & 0.94 & 0.09 & 0.87 \\
\hline Expression & & 0.06 & 0.26 & 0.94 & 0.11 & 0.19 & 0.93 & 0.13 & 0.88 \\
\hline Social interaction & & 0.23 & 0.84 & 0.24 & 0.2 & 0.82 & -0.06 & 0.13 & 0.82 \\
\hline Problem solving & & 0.24 & 0.79 & 0.31 & 0.1 & 0.87 & 0.25 & 0.26 & 0.83 \\
\hline Memory & & 0.19 & 0.82 & 0.19 & 0.02 & 0.85 & 0.21 & 0.21 & 0.83 \\
\hline
\end{tabular}

\section{Diagnoses}

(I) Vascular brain damage

(2) Brain injury

$(\mathrm{N}=337)$

(3) Post surgery

$(\mathrm{N}=27)$

$\quad(N=202)$

(4) Rheumatologic illness $\quad(\mathrm{N}=197)$

(5) Geriatric multiorgan illness $(\mathrm{N}=84)$

(6) other neurological ilness $(\mathrm{N}=192)$

\section{Factor analysis of $\mathbf{B l}$}

There is a well-documented discussion on the dimensionality of BI. Factor analytical and various multidimensional scaling methods were applied and reported. In order to analyze the factorial structure of BI we calculated factor analysis for various diagnosis groups with admission and discharge data. In all except vascular brain damage and traumatic brain injury groups we obtained more than the two factors which are prescribed as subscales explaining accounting for between 56 and $80 \%$ (Table 8 ). The BI mobility factor crystallised in all diagnostic groups (Getting in/out of bed, going to the toilet, bathing, walking, stairs climbing) but in addition to this we found also 'lower body dressing, 'personal hygiene' and 'washing' in all except in the post surgery and geriatric multiorgan illness group as a part of the first factor. Further, the first factor in some diagnosis

group also contained 'upper body dressing' (Table 9). The factorial structure of the vascular brain illness group consists of the firsts factor shared by other diagnostic groups, the second factor containing 'drinking', 'eating', 'bladder control' and 'bowel control'. The BI of the post surgery patients indicates a four factorial structure. First factor containing mobility items, second self care, third 'eating', 'drinking' and 'upper body dressing' and fourth 'bladder' and 'bowel control'. The rheumatological illness patients relate motor and self care items in factor 1, 'eating', 'upper body dressing', 'bowel' and 'bladder control' in factor 2 from which 'drinking' is separated in factor 3. The BI data of the geriatric multiorgan illness patients indicate a 4 factor structure with motor items (factor 1), self-care, 'dressing' and 'eating' (factor 2), 'bowel' and 'bladder control' (factor 3 ) and 'drinking' (factor 4). The factor analysis of the discharge data 
seems to fit to a similar factorial structure as the admission data. The vascular brain illness patients have a nearly identical factor 1 . The bladder and bowel control build a separate factor in discharge data, as do the items 'drinking' and 'eating'. The traumatic brain injury patients fit to 3 dimensions instead of 2 as on admission. The motor and self-care items need two dimension and bowel and bladder control build one factor._The following picture could be found in the group 'other neurological illness' (some mobility items with self care (F1), bladder and bowel control (F2), eating and drinking (F3). In the geriatric multiorgan illness group the items 'bladder' and 'bowel control', together with 'going to the toilet' are related to each other building one factor in the discharge data, while the motor and self care items distributed over two factors. Thus, we obtain a 2-4 factorial structure, depending on diagnosis and on the fact whether they are admission or discharge data.

Table 8 Factor analysis BI ( 13 items)

\begin{tabular}{|c|c|c|c|c|c|c|}
\hline \multicolumn{4}{|c|}{ Admission } & \multicolumn{3}{|c|}{ discharge } \\
\hline Fact. & E.VAL & $\begin{array}{l}\text { VAR } \\
\text { CUM }\end{array}$ & РCT & E.VAL & $\begin{array}{l}\text { VAR } \\
\text { CUM }\end{array}$ & PCT \\
\hline \multicolumn{7}{|c|}{ All patients } \\
\hline I & 6.06 & 46.6 & 46.6 & 5.99 & 46.1 & 46.1 \\
\hline 2 & 1.37 & 10.6 & 57.2 & 1.19 & 9.2 & 55.2 \\
\hline 3 & 1.07 & 8.2 & 65.4 & 1.11 & 8.5 & 63.7 \\
\hline \multicolumn{4}{|c|}{ 1) Vascular brain damage } & \multicolumn{3}{|c|}{$(\mathrm{N}=337)$} \\
\hline I & 6.44 & 49.6 & 49.6 & 6.53 & 50.2 & 50.2 \\
\hline 2 & 1.45 & 11.2 & 60.7 & 1.2 & 9.3 & 59.5 \\
\hline 3 & & & & 1.06 & 8.1 & 67.6 \\
\hline \multicolumn{4}{|c|}{ (3) Post surgery } & \multicolumn{3}{|c|}{$(\mathrm{N}=202)$} \\
\hline 1 & 4.45 & 34.3 & 34.3 & 5.11 & 39.3 & 39.3 \\
\hline 2 & 1.75 & 13.5 & 47.7 & 1.53 & 11.8 & 51.1 \\
\hline 3 & 1.19 & 9.2 & 56.9 & 1.26 & 9.7 & 60.7 \\
\hline 4 & 1.13 & 8.7 & 65.6 & & & \\
\hline
\end{tabular}

\begin{tabular}{lllllll}
\hline \multicolumn{3}{l}{ (4) Rheumatologic illness } & & \multicolumn{3}{c}{$(\mathbf{N}=197)$} \\
\hline I & 5.67 & 43.6 & 43.6 & 5.56 & 46.4 & 46.4 \\
2 & 1.29 & 9.9 & 53.6 & 1.35 & 11.2 & 57.6 \\
3 & 1.04 & 8 & 61.6 & & &
\end{tabular}

\begin{tabular}{llllllll}
\hline (5) & Geriatric & multiorgan illness & \multicolumn{3}{l}{$(\mathbf{N}=\mathbf{8 4})$} & & \\
\hline 1 & 5 & 38.5 & 38.5 & 4.39 & 33.7 & 33.7 \\
2 & 1.5 & 11.5 & 50 & 1.7 & 13.1 & 46.8 \\
3 & 1.21 & 9.3 & 59.3 & 1.15 & 8.9 & 55.7 \\
4 & 1.05 & 8.1 & 67.4 & & & \\
\hline
\end{tabular}

\begin{tabular}{lllllll}
\hline \multicolumn{2}{l}{ (6) other neurological ilness } & \multicolumn{3}{l}{$(\mathbf{N}=1$ 92) } \\
\hline 1 & 6.44 & 49.5 & 49.5 & 6.21 & 47.8 & 47.8 \\
2 & 1.3 & 10 & 59.5 & 1.58 & 12.2 & 60 \\
3 & 1.19 & 9.1 & 68.7 & 1.04 & 8 & 68 \\
\hline
\end{tabular}

Citation:Valach L, Selz B, Hofer K, et al.A six-year audit in a rehabilitation centre with mod barthel index and FIM as outcome measures. Int Phys Med Rehab J. 2018;3(4):342-358. DOI: I0.15406/ipmrj.2018.03.00I28
Table 9 Factor analysis BI (total).

\begin{tabular}{|c|c|c|c|c|c|c|}
\hline \multirow{2}{*}{$\begin{array}{l}\text { All } \\
(N=1039)\end{array}$} & \multicolumn{3}{|c|}{ admission } & \multicolumn{3}{|c|}{ discharge } \\
\hline & Fact I & Fact 2 & Fact 3 & Fact I & Fact 2 & $\begin{array}{l}\text { Fact } \\
3\end{array}$ \\
\hline Drinking & 2 & $.61 *$ & 0.42 & .06 & 14 & $.83 *$ \\
\hline Eating & 0.14 & $.76^{*}$ & 0.13 & 0.24 & -0.03 & $.77^{*}$ \\
\hline $\begin{array}{l}\text { Upper } \\
\text { body } \\
\text { dressing }\end{array}$ & 0.42 & $.67 *$ & 0.09 & $.59 *$ & 0.24 & 0.32 \\
\hline $\begin{array}{l}\text { Lower } \\
\text { body } \\
\text { dressing }\end{array}$ & $.69 *$ & 0.42 & 0.11 & $.79 *$ & 0.21 & 0.11 \\
\hline $\begin{array}{l}\text { Personal } \\
\text { hygiene }\end{array}$ & $.57^{*}$ & 0.47 & -0.03 & $.61 *$ & 0.15 & 0.29 \\
\hline Washing & $.70^{*}$ & 0.36 & 0.02 & $.74^{*}$ & 0.13 & 0.14 \\
\hline $\begin{array}{l}\text { Bladder } \\
\text { control }\end{array}$ & 0.28 & 0.13 & $.8 I^{*}$ & 0.29 & $.82 *$ & 0.06 \\
\hline $\begin{array}{l}\text { Bowel } \\
\text { control }\end{array}$ & 0.17 & 0.19 & $.83^{*}$ & 0.2 & $.88^{*}$ & 0.08 \\
\hline $\begin{array}{l}\text { Getting in/ } \\
\text { out of bed }\end{array}$ & $.74^{*}$ & 0.26 & 0.24 & $.7 I^{*}$ & 0.29 & 0.14 \\
\hline Toilet & $.77^{*}$ & 0.25 & 0.29 & $.75^{*}$ & 0.33 & 0.14 \\
\hline Bathing & $.74^{*}$ & 0.08 & 0.07 & $.66^{*}$ & 0.06 & 0.05 \\
\hline Walking & $.78 *$ & 0.05 & 0.25 & $.78^{*}$ & 0.17 & 0.1 \\
\hline Stairs & $.83^{*}$ & 1 & 0.23 & $.81 *$ & 0.13 & 0.08 \\
\hline
\end{tabular}

\begin{tabular}{|c|c|c|c|c|c|}
\hline \multirow{2}{*}{$\begin{array}{l}\text { (I) Vascular brain } \\
\text { damage }(\mathrm{N}=337)\end{array}$} & \multicolumn{2}{|c|}{ admission } & \multicolumn{3}{|c|}{ discharge } \\
\hline & fact I & fact 2 & fact I & fact 2 & fact 3 \\
\hline Drinking & 0.04 & $.72^{*}$ & 0.06 & 0.25 & $.84 *$ \\
\hline Eating & 0.3 & $.44^{*}$ & 0.34 & -0.11 & $.70 *$ \\
\hline Upper body dressing & $.76 *$ & 0.18 & $.69 *$ & 0.32 & 0.16 \\
\hline Lower body dressing & $.8 I^{*}$ & 0.21 & $.84 *$ & 0.21 & 0.12 \\
\hline Personal hygiene & $.69 *$ & 0.16 & $.60^{*}$ & 20 & 0.19 \\
\hline Washing & $.76 *$ & 0.19 & $.75^{*}$ & 0.17 & 0.1 \\
\hline Bladder control & 0.27 & $.73^{*}$ & 0.32 & $.82^{*}$ & -0.04 \\
\hline Getting in/out of bed & $.84 *$ & 0.21 & $.77^{*}$ & 0.21 & 0.16 \\
\hline Toilet & $.83 *$ & 0.3 & $.77^{*}$ & 0.34 & 0.2 \\
\hline Bathing & $.66^{*}$ & 0.08 & $.70 *$ & 0.03 & 0.01 \\
\hline Walking & $.79 *$ & 0.24 & $.8 I^{*}$ & 0.15 & 0.22 \\
\hline Stairs & $.8 I^{*}$ & 0.21 & $.85^{*}$ & 0.15 & 0.17 \\
\hline
\end{tabular}

\begin{tabular}{lccccc}
\multirow{2}{*}{$\begin{array}{c}\text { (2) Brain injury } \\
(\mathbf{N}=27)\end{array}$} & \multicolumn{2}{c}{ admission } & \multicolumn{3}{c}{ discharge } \\
\cline { 2 - 6 } & fact I & fact 2 & fact I & fact 2 & fact 3 \\
\hline Drinking & $.92^{*}$ & 0.14 & & & \\
Eating & 0.52 & $.60^{*}$ & 0.12 & -0.21 & $.94^{*}$ \\
Upper body dressing & $.87^{*}$ & 0.29 & 0.6 & $.64^{*}$ & 0.08 \\
Lower body dressing & $.69^{*}$ & $.67^{*}$ & $.86^{*}$ & 0.01 & 0.4 \\
Personal hygiene & $.67^{*}$ & 0.53 & 0.33 & 0.31 & $.80^{*}$
\end{tabular}




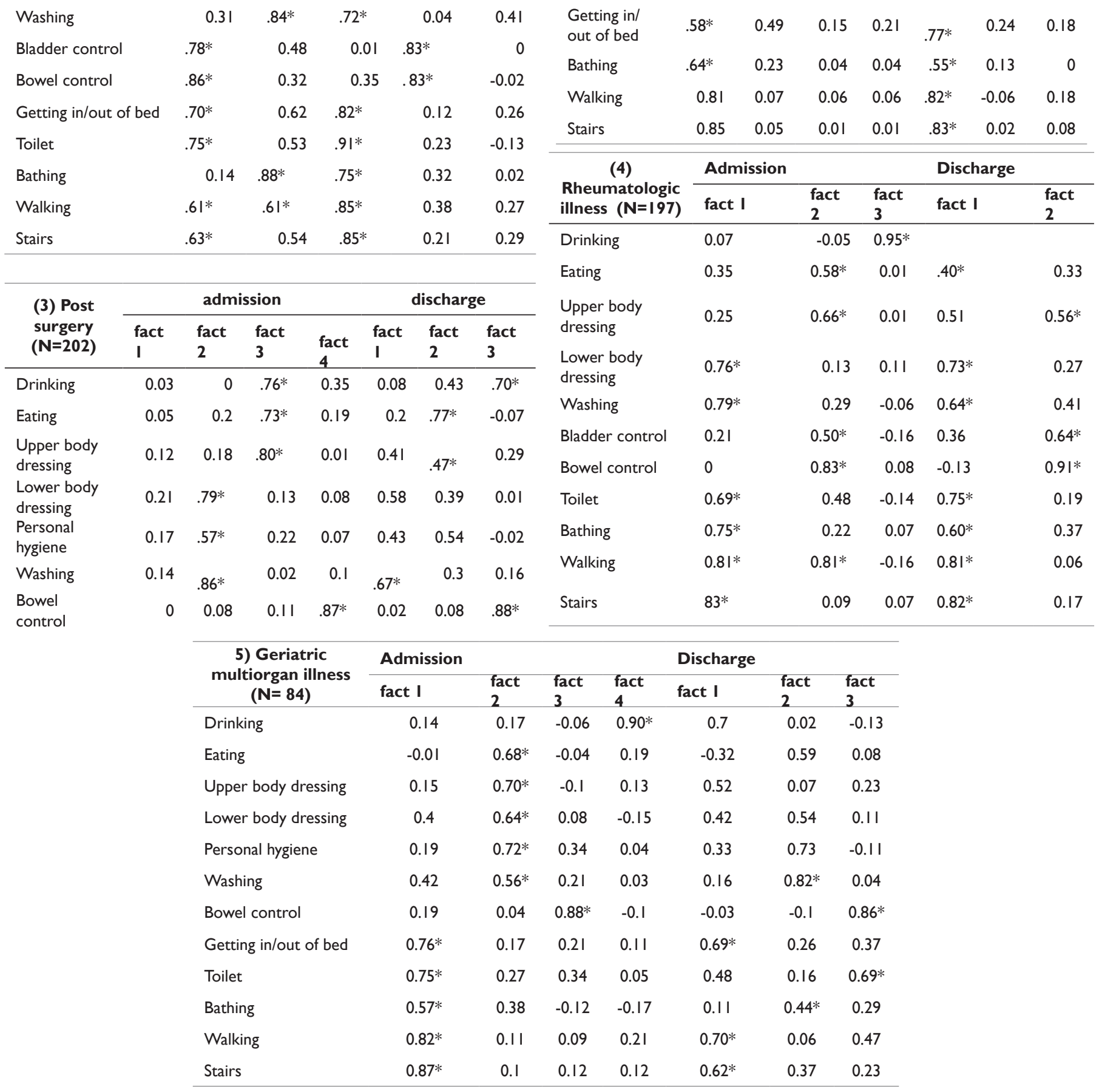

\section{Reliability}

The three Barthel scales (total, self care, mobility) reached satisfactory reliability in all patient groups on admission and on discharge (Table 10). Although the lowest reliability coefficient $(0.60$ in rheumatological illness, on discharge, self care scale) would call for improvement the next lowest reliability coefficients $(0.69$ in post surgery patients on admission, self care scale; other reaching 0.70 ) are acceptable. In most cases the reliability coefficients of admission data are very similar to those of discharge data within each of the patient groups.
The reliability coefficients of FIM scales (total, motor, cognitive) are generally higher (the lowest being 0.80 (in geriatric multiorgan illness patients on discharge, cognitive scale) (out of 42 reliability coefficients are only 9 between .80 and .89 . The other are equal or higher than .90) (Table 10).

\section{Comparison of scale means on admission and discharge}

BI

Analysing whether the patients' BI scores improved between 
admission and discharge we calculated $t$ test for dependent samples for each diagnosis group (Table 11). There is a highly significant improvement in the mean values of the BI total (sum of all items) in all groups $(p<0.001)$ except in patients with brain injury $(p<0.05)$. The mean values of the BI self care scale are significantly higher on discharge than on admission in all patients group $(\mathrm{p}<0.001)$ except in patients with traumatic brain injury $(\mathrm{p}<0.06)$. The differences in $\mathrm{BI}$ mobility scale between admission and discharge are also significant (traumatic brain injury patients: $\mathrm{p}<0.05$; all other groups $\mathrm{p}<0.001$ ) (Table 11).

Table I0 Reliability coefficient of barthel and fim scales.

\begin{tabular}{|c|c|c|c|c|c|c|c|}
\hline Diagnosis & I & 2 & 3 & 4 & 5 & 6 & all \\
\hline \multicolumn{8}{|c|}{ Barthel sum all } \\
\hline admission & 0.87 & 0.94 & 0.77 & 0.84 & 0.82 & 0.87 & 0.86 \\
\hline discharge & 0.88 & 0.87 & 0.82 & 0.84 & 0.79 & 0.87 & 0.86 \\
\hline \multicolumn{8}{|c|}{ Barthel self care } \\
\hline admission & 0.79 & 0.92 & 0.69 & 0.72 & 0.7 & 0.81 & 0.78 \\
\hline discharge & 0.79 & 0.7 & 0.7 & 0.77 & 0.6 & 0.8 & 0.77 \\
\hline \multicolumn{8}{|c|}{ Barthel motor } \\
\hline admission & 0.83 & 0.84 & 0.72 & 0.77 & 0.78 & 0.79 & 0.8 \\
\hline discharge & 0.83 & 0.86 & 0.78 & 0.72 & 0.78 & 0.78 & 0.8 \\
\hline \multicolumn{8}{|c|}{ FIM sum all items } \\
\hline admission & 0.96 & 0.98 & 0.94 & 0.95 & 0.93 & 0.95 & 0.96 \\
\hline discharge & 0.96 & 0.96 & 0.94 & 0.95 & 0.93 & 0.95 & 0.96 \\
\hline \multicolumn{8}{|c|}{ FIM motor } \\
\hline admission & 0.97 & 0.98 & 0.94 & 0.96 & 0.94 & 0.96 & 0.96 \\
\hline discharge & 0.97 & 0.97 & 0.93 & 0.96 & 0.95 & 0.97 & 0.96 \\
\hline \multicolumn{8}{|c|}{ FIM cognitiv } \\
\hline admission & 0.88 & 0.97 & 0.89 & 0.88 & 0.83 & 0.92 & 0.9 \\
\hline discharge & 0.88 & 0.93 & 0.89 & 0.86 & 0.8 & 0.91 & 0.89 \\
\hline
\end{tabular}

Diagnoses

(I) Vascular brain damage $\quad(\mathrm{N}=337)$

(2) Brain injury $\quad(\mathrm{N}=27)$

(3) Post surgery $\quad(\mathrm{N}=202)$

(4) Rheumatologic illness $\quad(\mathrm{N}=197)$

(5) Geriatric multiorgan illness $(\mathrm{N}=84)$

(6) other neurological iness $(\mathrm{N}=192)$

Table I I Barthel scales admission vs discharge (T-test for dependent samples)

\begin{tabular}{|c|c|c|c|c|c|c|c|c|}
\hline \multirow{2}{*}{ scale } & \multicolumn{3}{|c|}{ admission } & \multicolumn{5}{|c|}{ discharge } \\
\hline & mean & stddev & std err & $\begin{array}{c}\text { mean } \\
(\mathrm{N}=326)\end{array}$ & stddev & std err. & $\mathbf{t}$ & $\mathbf{p}$ \\
\hline \multicolumn{9}{|c|}{ (I) Vascular brain damage $(\mathrm{N}=326)$} \\
\hline All items & 73.17 & 25.815 & 1.43 & 85.54 & 20.827 & 1.153 & -12.4 & 0 \\
\hline Self care & 41.94 & 12.15 & 0.673 & 46.81 & 9.608 & 0.532 & -9.73 & 0 \\
\hline Mobility & 31.24 & 15.537 & 0.861 & 38.73 & 12.522 & 0.694 & -11.72 & 0 \\
\hline \multicolumn{9}{|c|}{ (2) Brain injury $\quad(N=27)$} \\
\hline All items & 80.93 & 30.368 & 5.844 & 92.7 & 14.982 & 2.883 & -2.24 & 0.034 \\
\hline Self care & 43.59 & 16.049 & 3.089 & 49.19 & 7.174 & 1.381 & -1.99 & 0.057 \\
\hline Mobility & 37.33 & 14.705 & 2.83 & 43.52 & 8.763 & 1.687 & -2.4 & 0.024 \\
\hline \multicolumn{9}{|c|}{ (3) Post surgery ( $\mathrm{N}=199)$} \\
\hline All items & 70.9 & 20.137 & 1.428 & 88.32 & 16.168 & 1.146 & -14.11 & 0 \\
\hline Self care & 43.13 & 9.364 & 0.664 & 48.67 & 6.881 & 0.488 & -9.55 & 0 \\
\hline Mobility & 27.77 & 13.795 & 0.978 & 39.65 & 11.025 & 0.782 & -13.8 & 0 \\
\hline
\end{tabular}


Table Continued

\begin{tabular}{|c|c|c|c|c|c|c|c|c|}
\hline \multirow{2}{*}{ scale } & \multicolumn{3}{|c|}{ admission } & \multicolumn{5}{|c|}{ discharge } \\
\hline & mean & stddev & std err & $\begin{array}{c}\text { mean } \\
(\mathrm{N}=326)\end{array}$ & stddev & std err. & $\mathbf{t}$ & $\mathbf{p}$ \\
\hline All items & 89.1 & 17.063 & 1.228 & 93.61 & 13.29 & 0.957 & -5.89 & 0 \\
\hline Self care & 48.61 & 7.314 & 0.526 & 49.88 & 7.056 & 0.508 & -3.23 & 0.001 \\
\hline Mobility & 40.49 & 10.885 & 0.784 & 43.73 & 7.441 & 0.536 & -6.43 & 0 \\
\hline
\end{tabular}

(5) Geriatric multiorgan illness ( $N=84)$

$\begin{array}{lrrrrrrrrr}\text { All items } & 73.1 \mathrm{I} & 22.19 \mathrm{I} & 2.45 \mathrm{I} & 84.78 & 16.797 & 1.855 & -6.29 & 0 \\ \text { Self care } & 42.34 & 9.964 & \mathrm{I} .1 & 45.62 & 8.187 & 0.904 & -3.72 & 0 \\ \text { Mobility } & 30.77 & 14.69 & 1.622 & 39.16 & 10.914 & 1.205 & -6.29 & 0\end{array}$

(6) other neurological ilness $(\mathrm{N}=190)$

\begin{tabular}{lrrrrrrrr} 
All items & 72.23 & 26.6 & 1.93 & 80.26 & 24.51 & 1.778 & -8.34 & 0 \\
Self care & 40.9 & 13.296 & 0.965 & 44.5 & 11.975 & 0.869 & -6.86 & 0 \\
Mobility & 31.33 & 15.338 & 1.113 & 35.76 & 14.256 & 1.034 & -7.23 & 0 \\
All patients & $\mathbf{( N = 1 0 I 7 )}$ & & & & & & & \\
All items & 75.78 & 24.205 & 0.759 & 86.76 & 19.547 & 0.613 & -21.05 & 0 \\
Self care & 43.32 & 11.354 & 0.356 & 47.29 & 9.22 & 0.289 & -15.43 & 0 \\
Mobility & 32.46 & 14.878 & 0.467 & 39.47 & 11.842 & 0.371 & -20.5 & 0 \\
\hline
\end{tabular}

\section{FIM}

A comparison of FIM total means on admission and discharge indicates a significant improvement of all patient groups (patients with traumatic brain injury: $\mathrm{p}<0.005$; all other groups: $\mathrm{p}<0.001$ ) (Table 12). The lowest FIM total mean was achieved by the vascular brain disease patients ( 83 points on admission) and the highest by the rheumatological patients (115 points on discharge). The smallest difference between admission and discharge FIM total can be find in rheumatological patients (5 points), the largest in patients with vascular brain disease (14 points) and in traumatic brain injury patients (15 points). The FIM motor scale indicates highly significant changes in all groups of patients $(\mathrm{p}<0.001)$ with the exception of traumatic brain injury patients $(p=0.008)$ (Table 12). The FIM cognitive admission - discharge comparison shows significant changes in vascular brain disease, in post surgical patients and in patients with other neurological illness $(\mathrm{p}<0.001)$. Patients with rheumatological illness $(p=0.013)$, patients with traumatic brain injury $(p=0.057)$ and geriatric multiorgan illness patients $(p=0.424)$ achieved less noticeable improvement (Table 12).

Table 12: Comparisson of FIM (admission) and FIM (discharge) (FIM total (sum), FIM motor and FIM cognitive (mean))

\begin{tabular}{llllll}
\hline \multicolumn{2}{l}{ admission } & \multicolumn{3}{c}{ discharge } \\
\cline { 2 - 3 } mean stddev mean & stddev $t$ & $\mathbf{p}$
\end{tabular}

(I) vascular brain

$\begin{array}{lrrrrrr}\text { FIM total } & 82.92 & 27.716 & 97.12 & 24.847 & -16.61 & 0\end{array}$

$\begin{array}{lllllll}\text { FIM motor } & 4.45 & \mathrm{I} .754 & 5.38 & \mathrm{I} .567 & -16.39 & 0\end{array}$

$\begin{array}{lllllll}\text { FIM } & 5.14 & 1.385 & 5.5 \mathrm{I} & \mathrm{I} .244 & -9.58 & 0\end{array}$

(2) traumatic brain

injury $(\mathrm{N}=27)$

$\begin{array}{lllllll}\text { FIM total } & 92.44 & 31.41 & 108.15 & 19.108 & -3.1 & 0.005\end{array}$

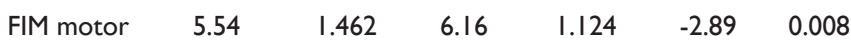

FIM

cognitive

5.23

$1.66 \mathrm{I}$

5.66

$1.403-2$

0.057

(3) post surgery

patients $(\mathrm{N}=202)$

$\begin{array}{lllllll}\text { FIM total } & 88.11 & 21.708 & 101.91 & 18.408 & -15.2 & 0\end{array}$

$\begin{array}{lllllll}\text { FIM motor } & 4.54 & \mathrm{I} .386 & 5.54 & \mathrm{I} .143 & -\mathrm{I} 5.92 & 0\end{array}$

FIM

cognitive $\quad 5.83$

(4) rheumatologica

illness $(\mathrm{N}=197)$

$\begin{array}{lllllll}\text { FIM total } & 109.83 & 18.853 & \text { II4.93 } & \text { I5.757 } & -7.42 & 0\end{array}$

$\begin{array}{lllllll}\text { FIM motor } & 6.03 & \mathrm{I} .255 & 6.38 & \mathrm{I} .035 & -7.09 & 0\end{array}$

$\begin{array}{lllllll}\text { FIM } & 6.29 & 0.96 & 6.39 & 0.869 & -2.5 & 0.013\end{array}$

(5) geriatric multiorgan

illness $(\mathrm{N}=84)$

$\begin{array}{lllllll}\text { FIM total } & 84.82 & 21.607 & 95.13 & 19.76 & -6.57 & 0\end{array}$

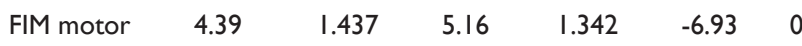

$\begin{array}{lllllll}\text { FIM } & 5.55 & \mathrm{I} .179 & 5.6 \mathrm{I} & \mathrm{I} .017 & -0.8 & 0.424\end{array}$

(6) other neurological

illnesses $(\mathrm{N}=192)$

$\begin{array}{lcccccc}\text { FIM total } & 86.8 & 26.814 & 95.9 & 25.568 & -10.13 & 0 \\ \text { FIM motor } & 4.6 & 1.754 & 5.21 & 1.703 & -9.98 & 0 \\ \text { FIM } & 5.48 & 1.46 \mathrm{I} & 5.7 \mathrm{I} & \mathrm{I} .304 & -4.62 & 0\end{array}$

Barthel total and FIM total score on discharge

FIM indicates in all diagnosis groups a higher proportion of improved patients.

A lower amount of patients indicate improvement in comparison with the observation with FIM.

(I) Vascular brain damage $(\mathrm{N}=337)$

(2) Brain injury $\quad(\mathrm{N}=27)$

(3) Post surgery $\quad(\mathrm{N}=202)$ 
(4) Rheumatologic illness $\quad(\mathrm{N}=197)$

(5) Geriatric multiorgan illness $(\mathrm{N}=84)$

(6) other neurological ilness $(\mathrm{N}=\mid 92)$

\section{Changes in BI and FIM}

The mean comparisons indicate mean changes in the whole groups of patients. However, the declared goal of a rehabilitation team is to help every patient. The information how many patients improved their total score on discharge is an important information on how many patients were judged as more independent on discharge and how many patients judged themselves as being more independent. The table 13 summarizes the data for each diagnostic group of patients. Well over three quarter of patients improved their independence as assessed by the nurses (FIM total) (minimum 64 maximum $87 \%$ of all patients of each diagnostic group; $80 \%$ of the whole sample). Some $10 \%$ did not change in their overall score $(5-28 \%), 10 \%$ did not manage to maintain their admission degree of independence $(7-17 \%)$. The BI scores indicate that patients assess their degree of improvement in their independence more sceptical than the nursing staff ( $61 \%$ patients vs. $80 \%$ of nurses indicate a change with discharge score higher than admission score). Less that a half of all brain injury patients and rheumatological patients report improvement in their independence ( $48 \%$ and $40 \%$ ). Building a group of patients who did change neither in BI nor in FIM we obtained about $12 \%$ of patients (whole sample; ranging from 26\% (rheumatological illness) to $6 \%$ (post surgery)) (Table 14). Who are these patients who did not improve their independence? Are they too severe cases (floor effect) or are they patients with a high admission score (ceiling effect)? (Table 13) (Table 14).

Table 13 Changes in Barthel total and FIM total score on discharge in comparisson to admission (patients in \%):

worse unchanged improved improved by 20BI/25FIM

All patients $(\mathrm{N}=1039)$

$\begin{array}{lllll}\text { BI } & 9 & 30 & 61 & 25 \\ \text { FIM all } & 9 & 1 \mathrm{I} & 80 & 15\end{array}$

(I) Vascular brain damage $(\mathrm{N}=337)$

$\begin{array}{lllll}\text { BI } & 12 & 25 & 63 & 27 \\ \text { FIM } & 8 & 5 & 87 & 22\end{array}$

(2) Brain injury $(\mathrm{N}=27)$

$\begin{array}{lllll}\text { BI } & 4 & 48 & 48 & 15 \\ \text { FIM } & 11 & 11 & 78 & 18\end{array}$

(3) Post surgery $\quad(\mathrm{N}=202)$

$\begin{array}{lllll}\text { BI } & 5 & 16 & 80 & 40 \\ \text { FIM } & 7 & 6 & 87 & 18\end{array}$

(4) Rheumatologic illness $\quad(\mathrm{N}=197)$

$\begin{array}{lllll}\mathrm{BI} & 7 & 53 & 40 & 10\end{array}$

$\begin{array}{lllll}\text { FIM } & 8 & 28 & 64 & 5\end{array}$

(5) Geriatric multiorgan illness

$\begin{array}{lllll}\mathrm{BI} & 15 & 17 & 68 & 28\end{array}$

$\begin{array}{lllll}\text { FIM } & 17 & 5 & 78 & 16\end{array}$

(6) other neurological ilness

$\begin{array}{lllll}\text { BI } & 12 & 31 & 57 & 27 \\ \text { FIM } & 10 & 12 & 78 & 8\end{array}$

Table I 4 Improvement between admission and discharge either in FIM total or in $\mathrm{BI}$ total

\begin{tabular}{llll}
\hline & \multicolumn{3}{l}{ improved } \\
\cline { 2 - 4 } & $\mathbf{N}$ & $\mathbf{N}$ all & $\%$ \\
\hline All & 1017 & 892 & 87.7 \\
vascular brain dissease & 326 & 304 & 93.3 \\
traumatic brain injury & 27 & 23 & 85.2 \\
post surgery & 199 & 187 & 94 \\
rheumatological illness & 193 & 142 & 73.6 \\
geriatric multiorgan illness & 82 & 74 & 90.2 \\
other neurological illnesses & 190 & 162 & 85.3 \\
\hline
\end{tabular}

A comparison of means of the patients who improved and those who did not in regards to the BI total, BI self care, BI mobility, FIM total, FIM motor, FIM cognitive, indicates that patients who did not improve their independence were more independent on admission that the patents who improved their independence (Table 15). Although this result is significant in the total sample there are some difference in the diagnostic groups. The result is not significant in vascular brain damage patients and in brain injury patients, where it is due to the low number of patients $(\mathrm{N}=4)$. In addition, there are some differences between BI being significant in post surgery patients and FIM being significant in 'other neurological illness' group. The results of this comparison would support the thesis that non-improvement of the patients is mostly due to the high admission values, that is, to the ceiling effect of the measures. However, it should be noted that all patients indicate a better value minimally in one of the BI items on discharge, thought some of them also report a worse value thus reaching a total which is either negative or unchanged (Table 15).

Table 15 Comparison (t-test) of patients with and without improvement in scales on admission.

\begin{tabular}{|c|c|c|c|c|c|c|}
\hline All patients Scales & no improv. Mean & $(N=125)$ stddev & improv. Mean & $(\mathrm{N}=892)$ stdden & $\mathbf{t}$ & $\mathbf{p}$ \\
\hline I Bl total & 87.18. & 22.256 & 74.18 & 24.008 & 5.99 & 0 \\
\hline $2 \mathrm{BI}$ self care & 48.17 & 10.284 & 42.64 & II.337 & 5.26 & \\
\hline 3 BI mobility & 39.02 & $|3.52|$ & 31.54 & 14.835 & 5.72 & \\
\hline 4 FIM total & 106.69 & 26.38 & 88.16 & 25.255 & 7.39 & \\
\hline 5 FIM motor (mean) & 5.84 & 1.742 & 4.66 & 1.64 & 7.14 & \\
\hline 6 FIM cognitiv (mean) & 6.14 & 1.165 & 5.5 & 1.368 & 5.6 & \\
\hline
\end{tabular}

Vascular brain damage 
Table Continued

\begin{tabular}{|c|c|c|c|c|c|c|}
\hline All patients Scales & no improv. Mean & $(N=125)$ stddev & improv. Mean & $(\mathrm{N}=892)$ stdden & $\mathbf{t}$ & $\mathbf{p}$ \\
\hline Scales & no improv. Mean & $(N=22)$ stddev & improv. Mean & $(\mathrm{N}=304)$ stdden & $\mathbf{t}$ & $\mathbf{p}$ \\
\hline $\mathrm{I} \mathrm{Bl}$ total & 77.41 & 31.895 & 72.87 & 25.355 & 0.65 & 0.52 \\
\hline $2 \mathrm{BI}$ self care & 43.23 & $|4.93|$ & 41.85 & 11.949 & 0.42 & 675 \\
\hline $3 \mathrm{BI}$ mobility & 34.18 & 18.16 & 31.02 & 15.342 & 0.8 & 434 \\
\hline 4 FIM total & 90.82 & 34.099 & 82.88 & 26.833 & 1.07 & 297 \\
\hline 5 FIM motor (mean) & 4.88 & 2.231 & 4.42 & $\mathrm{I} .725$ & 95 & 354 \\
\hline 6 FIM cognitiv (mean) & 5.48 & 1.408 & 5.09 & 1.396 & 1.26 & 220 \\
\hline \multicolumn{7}{|l|}{ Brain injury } \\
\hline Scales & no improv. Mean & $(N=4)$ stddev & improv. Mean & $(N=23)$ stdden & $\mathbf{t}$ & $\mathbf{p}$ \\
\hline $\mathrm{I} \mathrm{Bl}$ total & 90.25 & 14.66 & 79.3 & 32.281 & 1.1 & 0.299 \\
\hline $2 \mathrm{BI}$ self care & 48.75 & 4.924 & 42.7 & 17.187 & 1.39 & 0.181 \\
\hline $3 \mathrm{BI}$ mobility & 41.5 & 11 & 36.61 & $|5.34|$ & 0.77 & 0.475 \\
\hline 4 FIM total & 107.5 & 16.197 & 89.83 & 32.891 & 1.67 & 0.133 \\
\hline 5 FIM motor (mean) & 6.19 & 1.031 & 5.04 & 1.929 & 1.77 & 0.118 \\
\hline 6 FIM cognitiv (mean) & 5.4 & $\mathrm{I} .347$ & 4.87 & 1.992 & 0.67 & 0.529 \\
\hline \multicolumn{7}{|l|}{ Post surgery } \\
\hline Scales & no improv. Mean & $(N=12)$ stddev & improv. Mean & $(N=137)$ stdden & $\mathbf{t}$ & $\mathbf{p}$ \\
\hline I BI total & 84.83 & 18.566 & 70.01 & 19.949 & 2.67 & 0.02 \\
\hline $2 \mathrm{Bl}$ self care & 48.83 & 7.826 & 42.76 & 9.354 & 2.57 & 0.023 \\
\hline $3 \mathrm{BI}$ mobility & 36 & 13.239 & 27.24 & 13.695 & 2.22 & 0.046 \\
\hline 4 FIM total & 94.67 & 31.647 & 87.83 & 21.113 & 0.74 & 0.475 \\
\hline 5 FIM motor (mean) & 5.19 & 1.802 & 4.51 & 1.357 & 1.3 & 0.219 \\
\hline 6 FIM cognitiv (mean) & 5.43 & 1.831 & 5.85 & 1.116 & -0.78 & 0.449 \\
\hline \multicolumn{7}{|l|}{ Rheumatologic illness } \\
\hline Scales & no improv. Mean & $(N=52)$ stddev & improv. Mean & $(N=\mid 42)$ stdden & $\mathbf{t}$ & $\mathbf{p}$ \\
\hline $\mathrm{I} \mathrm{BI}$ total & 96.39 & 11.137 & 86.48 & 18.064 & 4.56 & 0 \\
\hline $2 \mathrm{BI}$ self care & 51.59 & 5.288 & 47.54 & 7.652 & 4.14 & 0 \\
\hline $3 \mathrm{BI}$ mobility & 44.8 & 6.779 & 38.94 & 11.658 & 4.3 & 0 \\
\hline 4 FIM total & 121 & 11.109 & 106.01 & 19.571 & 6.63 & 0 \\
\hline 5 FIM motor (mean) & 6.73 & 0.821 & 5.79 & 1.297 & 5.99 & 0 \\
\hline 6 FIM cognitiv (mean) & 6.69 & 0.579 & 6.16 & 1.021 & 4.5 & 0 \\
\hline \multicolumn{7}{|c|}{ Geriatric multiorgan illness } \\
\hline Scales & no improv. mean & $(\mathrm{N}=8)$ stddev & improv. Mean & $(N=74)$ stdden & $\mathbf{t}$ & $\mathbf{p}$ \\
\hline $\mathrm{I} \mathrm{BI}$ total & 89.5 & 10.406 & 71.34 & 22.437 & 4.03 & 0.001 \\
\hline $2 \mathrm{BI}$ self care & 50.63 & 4.502 & 41.45 & 9.995 & 4.66 & 0 \\
\hline $3 \mathrm{BI}$ mobility & 38.88 & 9.717 & 29.89 & $14.9 \mid 4$ & 2.33 & 0.04 \\
\hline 4 FIM total & 100.88 & 21.938 & 82.81 & 21.201 & 2.22 & 0.055 \\
\hline 5 FIM motor (mean) & 5.35 & 1.522 & 4.27 & 1.413 & 1.91 & 0.09 \\
\hline 6 FIM cognitiv (mean) & 6.28 & 0.667 & 5.46 & 1.212 & 2.96 & 0.011 \\
\hline \multicolumn{7}{|c|}{ other neurological ilness } \\
\hline Scales & no improv. Mean & $(N=28)$ stddev & improv. Mean & $(N=162)$ stdden & $\mathbf{t}$ & $\mathbf{p}$ \\
\hline $\mathrm{I} \mathrm{Bl}$ total & 78 & 28.191 & 71.23 & 26.278 & 1.18 & 0.244 \\
\hline
\end{tabular}


Table Continued

\begin{tabular}{lllllll} 
2 Bl self care & 44.75 & 13.094 & 40.23 & 13.257 & 1.68 & 0.101 \\
\hline All patients Scales & no improv. Mean & $\mathbf{( N = | 2 5 )}$ stddev & improv. Mean & $\mathbf{( N = 8 9 2 ) ~ s t d d e n ~}$ & $\mathbf{t}$ & $\mathbf{P}$ \\
\hline 3 BI mobility & 33.25 & 16.338 & 31 & 15.187 & 0.68 & 0.501 \\
4 FIM total & 99.79 & 28.026 & 84.98 & 25.913 & 2.61 & 0.013 \\
5 FIM motor (mean) & 5.35 & 1.996 & 4.45 & 1.697 & 2.25 & 0.031 \\
6 FIM cognitiv (mean) & 6.04 & 1.068 & 5.42 & 1.491 & 2.65 & 0.011 \\
\hline
\end{tabular}

\section{Discussion}

Our data provide some indication of the possible floor and ceiling effects of BI and FIM, though the lack of significant results on discharge in some of the patients is only partly due to ceiling or floor effects. Nevertheless, the only non significant result in Barthel Index was found in the group of the traumatic brain injury patients where also one of the highest number of patients with the highest Barthel self care score could be found. The non-significant admission-discharge comparisons of FIM scores are more difficult to associate with the floor and ceiling effects as the geriatric multiorgan illness patient do not reach exceptional floor or ceiling effect susceptible values. It could be argued that these patients although improving their motor functions do not improve enough their cognitive functions. Their cognitive functions are on admission better than in vascular brain disease patients, traumatic brain injury patients and other neurological illness patients. However, on discharge, although slightly improved, the rating of their cognitive functions is nearly the lowest of the 6 diagnoses group patients.

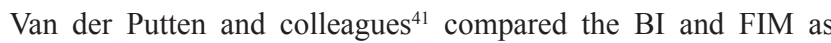
outcome measures after inpatient rehabilitation and report that BI and FIM total, FIM motor and FIM cognitive scales were appropriate measures for the stroke patients, but that FIM cognitive scale has limited usefulness in progressive multiple sclerosis because of its notable ceiling effect. We also found that FIM cognitive scale has limited usefulness for some diagnosis groups of patients such as geriatric multiorgan illness and traumatic brain injury patients, though the latter group is much too small for conclusive suggestions $(\mathrm{N}=27)$.

Laake and colleagues report that BI is unidimensional among stroke patients but not among geriatric patients or patients with hip fracture where one factor related to mobility and the other to bodily functions. Consequently, the sum score is not appropriate. An argument also forwarded by Sulter and co-workers.?

Kalra $^{48}$ compared BI of stroke patients managed in a stroke rehabilitation unit and in general wards. Although the initial Barthel score (median) was comparable between these two groups the median discharge Barthel score of patients managed in the stroke unit was significantly higher than of patients managed in general wards. There was a significant delay in discharging stroke patients in general wards (20 weeks) compared with those in the stroke unit (6 weeks). These and other similar results indicate that the improvements in our patient groups between admission and discharge cannot be attributed to 'spontaneous' recovery only. Consequently, it could be concluded that there was a significant improvement achieved in all patient groups in regard to independence in activities of daily living as measured by FIM and BI

As indicated at the beginning of this article measures integrated into a rehabilitation routine do not always reach the standards of a scientific double blind prospective control study. The assessing nurses or occupational therapists are aware of the fact whether the patient has just been admitted to the rehabilitation centre or he or she is going to be discharged. Despite all their working ethics and correct assessment they also are very much interested in patients' improvement although there are no negative sanction if it is not the case. The patients filling out $\mathrm{BI}$ are interested in going home and may not be always interested in indicating that their independence did not improve. These are but a few reservations one may have towards this data. However, if there were no improvement the patients would be deeply disappointed and may be interested in indicating how bad his or her situation is thus describing functional independence on discharge even lower than on the admission. Further, regular training of the nurses using FIM training video and discussing unclear cases and false assumptions the nurses may have helped in achieving certain coding standards which should limit the effect of wishful thinking. These would work against the assupmtions listed above.

The differences between BI and FIM as mirrored in our results could be inherent to the measure with FIM being more sensitive to change than the self-report BI. It is also is possible to assume that the changes were first manifested at a low level of action organisation thus being less obvious to the patients. Further, the often-reported depressive symptoms in rehabilitation patients could be responsible for theirs a more pessimistic view of their rehabilitation progress. Finally, it also should be discussed, whether nurses indicate change where there was not enough reason for seeing one. However, even the modest self-assessment shows over $60 \%$ of all patients indicating a change that is a result in support of the implemented programme. In many patients their given background for interpreting these results is not a stable condition or even a spontaneous improvement but a deterioration. Consequently, even maintenance of some patients' independence may be cosidered a success.

There may be a number of unsolved measurement and scaling problems in the assessment of the rehabilitation outcome. However, as some authors (such as the Rivermead team) pointed out, the contextual issues of independence should not be forgotten and will not disappear with better scaling methods.

Rehabilitation is a systematic joint goal directed process in which the patient is helped to gain more independence. This is partly the issue of functional independence but also to a very substantial part of the goal directed understanding and organisation of the patient's life. Consequently, actions, projects and goal directed careers be it an occupational, rehabilitational or any other socially embedded process must be monitored in their psychosocial anchoring. Psychology and social psychology offers a series of methods how to capture these processes. Concepts such as empowerment, self-efficacy, goal-directed action, action related cognitions and many other offer a good possibility of merging the functional independence with its psychological and social steering. The new ICIDH-2 classification may help us to redirect our empirical views. In consequently conceptualizing the rehabilitation process as a goal directed we have 
to utilize measures that are closely linked to the setting of the goal of the rehabilitation. Approaches such as 'goal-attainment scaling'. ${ }^{49}$.

\section{Acknowledgments}

None.

\section{Conflict of interest}

The author declares no conflict of interest.

\section{References}

1. Turner-Stokes L. Editorial. Clinical Rehabilitation, 1999;13:273-275.

2. Oremus M, Santaguida P, Walker K, et al. Methodological Issues in Evaluation of Innovative Training Approaches to Stroke Rehabilitation [Internet]. Rockville (MD): Agency for Healthcare Research and Quality (US); 2008.

3. Turner SL, Turner ST. The use of standardized outcome measures in rehabilitation centred in the UK. Clinical Rehabilitation, 1997;11(4):306313.

4. Mahoney FI, Barthel DW. Functional evaluation: the Barthel Index. Md State Med J. 1965; 14:61-65.

5. Hamilton BB, Granger CV, Sherwin FS. A uniform national data system for medical rehabilitation. In: JM Fuhrer, editor, Rehabilitation outcomes: analysis and measurement. 1987;137-47.

6. Hall KM, Hamilton BB, Gordon WA, et al. Characteristics and comparisons of functional assessment indices: Disability Rating Scale, Functional Independence Measure and Functional Assessment Measure. J Head Trauma Rehabil. 1993;8(2):60-74

7. Sulter G, Steen C, De Keyser J. Use of the Barthel Index and Modified Ranking Scale in acute stroke trials. Stroke, 1999;30(8):1538-1541.

8. Duffy L, Gajree S, Langhorne P, et al. Reliability (inter-rater agreement) of the Barthel Index for assessment of stroke survivors: systematic review and meta-analysis. Stroke. 2013;44(2):462-468.

9. Ganesh A, Gutnikov SA, Rothwell PM. Late functional improvement after lacunar stroke: a population-based study. J Neurol Neurosurg Psychiatry. 2018.

10. Hsieh CL, Hoffmann T, Gustafsson L, et al. The diverse constructs use of activities of daily living measures in stroke randomized controlled trials in the years 2005-2009. J Rehabil Med. 2012;44(9):720-726.

11. Prodinger B, O'Connor RJ, Stucki G, et al. Establishing score equivalence of the Functional Independence Measure motor scale and the Barthel Index, utilising the International Classification of Functioning, Disability and Health and Rasch measurement theory. J Rehabil Med. 2017;49(5):416-422.

12. Treweek SP, \& Condie ME. Three measures of functional outcome for lower limb amputees: a retrospective review. Prosthetics and Orthotics International. 1998;22(3):178-185.

13. Wellwood I, Dennis MS, \& Warlow CP. A comparison of the Barthe Index and the OPCS disability instrument used to measure outcome after acute stroke. Age Ageing. 1995;24(1):54-57.

14. Schonle PW. The early rehabilitation Barthel Index - an early rehabilitation oriented extension of the Barthel Index. Rehabilitation. 1995;34(2):69-73

15. Shah $\mathrm{S}$, Muncer $\mathrm{S}$. Outcome measurement in brain injury rehabilitation towards a common language. Clinical Rehabilitation, 2000;14:340-342.

16. Granger CV, Greer DS. Functional status measurement and medical rehabilitation outcomes. Archives of Physical Med. Rehabil. 1976;57(3):103-109.

17. Shah S, Vanclay F, Cooper C. Improving the sensitivity of the Barthel Index for stroke rehablitation. Journal of Clinical Epidemiology.
1989;42(8):403-709.

18. Hocking C, Williams M, Broad J, et al. Sensitivity of shah, vanclay and cooper's modified barthel index. Clinical Rehabilitation. 1999;13(2):141-147.

19. Law M, Letts L. A critical review of scales of activities of daily living. The American Journal of Occupational Therapy, 1989;43(8):522-528.

20. Collin C, Wade DT, Davies S, et al. The Barthel ADL Index: A reliability study. International Disability Studies, 1988;10(2):61-63.

21. Shah S, Cooper B. Commentary on 'a critical evaluation of the Barthel Index'. British Journal of Occupational Therapy, 1993;56(2):70-72.

22. Granger CV, Albrecht GL, Hamilton BB. Outcome of comprehensive medical rehabilitation: measurement by PULSES profile and the Barthel index. Arch. of Physical Med. Rehabil. 1979;60(4):145-154.

23. Wade DT, Langton HR. Functional abilities after stroke: measurement, natural history and prognosis. Journal of Neurology, Neurosurgery and Psychiatry. 1987;50(2):177-182.

24. Adams RJ, Meador KJ, Sethi KD, et al. Graded neurologic scale for use in acute hemispher stroke treatment protokols. Stroke, 1987;18(3):665669.

25. Granger CV, Greer DS, Liset E. Measurement of outcomes of care for stroke patients. Stroke. 1975;6:34-41

26. Hertanu JS, Demopoulos JT, Yang WC, et al. Stroke rehabilitation: Correlation and prognostic value of computerized tomography and sequential functional assessment. Archives of Physical Med. Rehabil. 1984;65(9):505-508.

27. Wood DSL, Williams JI, Shapiro SH. Examining outcome measures in a clinical study of stroke. Stroke. 1990;21(5):731-739.

28. Walker MF, Gladman JR, Lincoln NB, et al. Occupational therapy for stroke patients not admitted to hospital: a randomized controlled trial. The Lancet, 1999;354(9175):278-280.

29. Alarcon T, Bercena A, Gonzalez MJI, et al. Factor predictive of outcome on admission to an acute geriatric ward. Age Ageing, 1999;28(5):429 432.

30. Shinar D, Gross CR, Bronsteinm KS, et al. Reliability of the activities of daily living scale and its use in telephone interview. Archives of Physical. Med Rehabil, 1987;68(10):723-728.

31. Roy CW, Tognneri J, Hay E, et al. An inter-rater reliability study of the Barthel index. International Journal of Rehabilitation Research. 1988;11(1):67-70.

32. Ranhoff $\mathrm{AH}, \&$ Laake $\mathrm{K}$. The Barthel ADL index scoring by the physician from patient interview is not reliable. Age Ageing. 1993;22(3):171174.

33. Korner BN, Wood DS. Barthel Index information elicited over the telephone. Is it reliable? American Journal of Physical Med. Rehabil. 1995;74(1):9-18.

34. Yeo D, Faleiro R, Lincoln NB. Rathel ADL index: a comparison of administration methods. Clinical Rehabilitation. 1995;9:34-39.

35. McGinnis GE, Seward ML, DeJong G, et al. Program evaluation of physical medicine and rehabilitation departements using self-report Barthel. Archives of Physical Med. Rehabil. 1986;67(2):123-25.

36. Gompertz P, Pound P, Ebrahim S. A postal version of the Barthel Index. Clinical Rehabilitation. 1994;8(3):233-239.

37. Wade DT. Personal physical disability. In: Measurement in neurological rehabilitation. Oxford: Oxford Medical Publication. 1992.

38. Granger $\mathrm{CV}$, Cotter AC, Hamilton BB, et al.. Functional Assessment scales: a study of persons with multiple sclerosis. Arch Phys Med Rehabil. 1990;71(11):870-875. 
39. Heinemann AW, Linacre JM, Wright BD, et al. Relationship between impairment and physical disability as measured by the functional independence measure. Archives of Physical Medicine and Rehabilitation. 1993;74:566-573.

40. Chau N, Daler S, Andre JM, et al. Inter-rater agreement of two functional independence scales: the Functional Independence Measure (FIM) and the subjective uniform continuous scale. Disability and Rehabilitation. 1994;16(2):63-71.

41. Van der Putten JJ, Hobart JC, Freeman JA, et al. Measuring change in disability after inpatient rehabilitation: comarison of the responsiveness of the Barthel Index and the Functional Independence Measure. Journal of Neurology, Neurosurgery and Psychiatry, 1999;66(4):480-484.

42. Nyein K, McMichael L, Turner SL. Can a Barthel score be derived from the FIM?. Clinical Rehabilitation. 1999;13:56-63.

43. Granger CV, Sherwood CC, Greer DS. Functional status measures in a comprehensive stroke care program. Archives of Physical Med. Rehabil. 1977;58(12):555-561.

44. De Weerdt W, Selz B, Nuyens G, et al. Time use of stroke patients in a in- tensive rehabilitation unit: a comparison between a Belgian and a Swiss setting. Disability and Rehabilitation. 2000;22(4):181-186.

45. Feys HM, De Weerdt WJ, Selz BE, et al. Effect of a theraptic intervention for the hemiplegic upper limb in the acute phase after stroke. A single-blind, randomized, controlled multicenter trial. Stroke. 1998;29(4):785-792.

46. Ravaud JF, Delcey M, Yelnik A. Construct validity of the Functional Independence Measure (FIM): Questioning the unidimensionality of the scale and the "value of FIM scores. Scandinavian Journal of Rehabilitation Medicine. 1999;31(1):31-41.

47. Laake K, Laake P, Ranhoff AH, et al. The Barthel ADL Index: factor structure depends upon the category of patient. Age Ageing, 1995;24(5):393-397.

48. Kalra L. The influence of stroke unit rehabilitation on functional recovery from stroke. Stroke, 1994;25(4):821-825.

49. Kiresuk TJ, Smith A, Cardillo, editors. Goal attainment scaling. Hilsdale: Erlbaum. 1994. 